Portland State University

PDXScholar

7-8-1991

\title{
Computer-Based Parameter Visualization and Decision Making for the Detection and Prediction of Ovulation: the Ovulo-Computer
}

Omar Rodolfo Bermudez

Portland State University

Follow this and additional works at: https://pdxscholar.library.pdx.edu/open_access_etds

Part of the Electrical and Computer Engineering Commons Let us know how access to this document benefits you.

\section{Recommended Citation}

Bermudez, Omar Rodolfo, "Computer-Based Parameter Visualization and Decision Making for the Detection and Prediction of Ovulation: the Ovulo-Computer" (1991). Dissertations and Theses. Paper 4179.

https://doi.org/10.15760/etd.6063

This Thesis is brought to you for free and open access. It has been accepted for inclusion in Dissertations and Theses by an authorized administrator of PDXScholar. Please contact us if we can make this document more accessible: pdxscholar@pdx.edu. 
AN ABSTRACT OF THE THESIS OF Omar Rodolfo Bermudez for the Master of Science in Electrical and Computer Engineering presented July 8, 1991.

Title: Computer-based Parameter Visualization and Decision-making for the Detection and Prediction of Ovulation: The Ovulo-Computer.

APPROVED BY THE MEMBERS OF THE THESIS COMMITTEE:

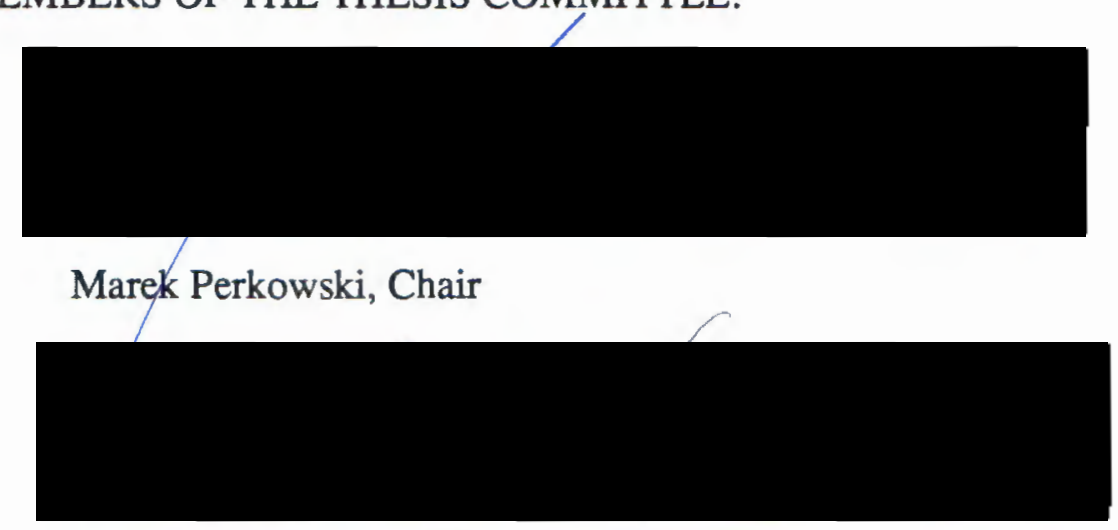

Michael A. Driscoll

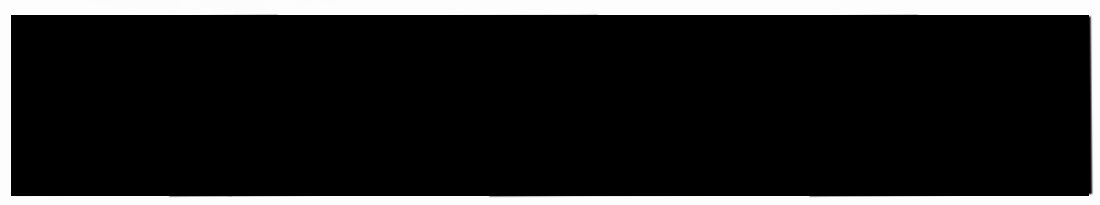

Rose Fuller

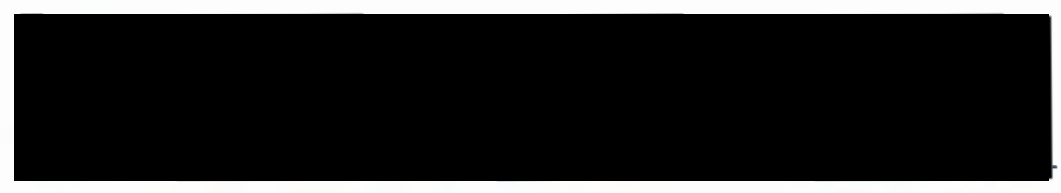

Warren Harrison

It is now well accepted by gynecologists that a woman can conceive from an act of intercourse for a maximum of seven days of her menstrual cycle [1]. The reliability of 
natural family planning methods depends on identifying this window of fertility without ambiguity. Several natural family planning (NFP) methods have been used extensively and with some success. For decades, cycle length and BBT history have been used to forecast the most likely days of ovulation. When greater precision is needed, serum LH assays or ultrasound techniques are used. With the recent development of enzyme-linked immunosorbent assay, various kits have become commercially available to provide a simple, self-administered, and rapid test for detection of the midcycle urinary LH surge [2]. Some of these methods are fairly reliable and accurate. However, failures in these methods still occur, and some other problems concerning accuracy, simplicity, cost, and comfort have not been solved yet [3]. In addition, other factors in NFP methods, such as the user, the procedure, and others, have been demonstrated to be important sources of errors.

The prototype system developed in this thesis, called the OVULO-COMPUTER (OC), deals with these unsolved issues by integrating the most well accepted methods of family planning in a computer-knowledge-base system capable of acquiring, manipulating, storing, and displaying data in real-time. In addition to monitoring ovulation, the $\mathrm{OC}$ also automates the process of rule comparison and evaluation by providing the physicians with results from different rules applied to the same set of data. The system consists of an IBM compatible, 286 or 386 microcomputer, an 8-channel analog-to-digital converter, an I/O interface program, and a main central program written in Lotus 1-2-3 Command Language. The software operates under MS-DOS operating system. New features of the 1-2-3 Release 3.1 allow improved user interface capabilities, as well as enhanced graphics presentation. The OC is a user-friendly system designed to help doctors and nurses make easier and more accurate clinical decisions in the analysis and interpretation of the fertility status in women. 
COMPUTER-BASED PARAMETER VISUALIZATION AND DECISION MAKING FOR THE DETECTION AND PREDICTION OF OVULATION:

THE OVULO-COMPUTER

\author{
by \\ OMAR RODOLFO BERMUDEZ
}

A thesis in partial fulfillment of the requirements for the degree of

\author{
MASTER OF SCIENCE \\ in \\ ELECTRICAL AND COMPUTER ENGINEERING
}

Portland State University

1991 


\section{TO THE OFFICE OF GRADUATE STUDIES:}

The members of the committee approve the thesis of Omar Rodolfo Bermudez presented July 8, 1991.

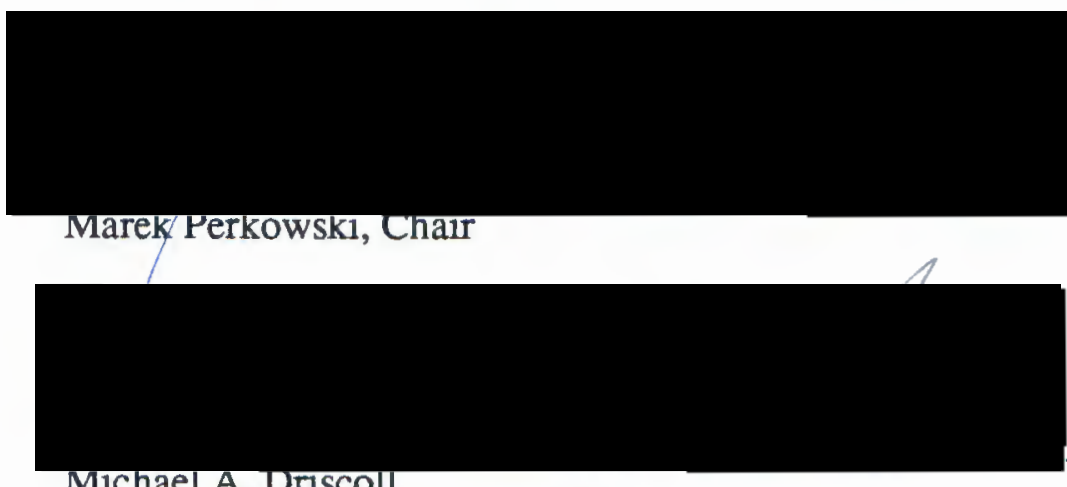

IVichael A. Driscoll

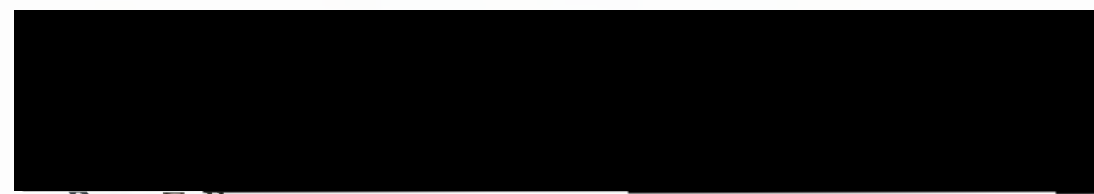

\section{Rose Fuller}

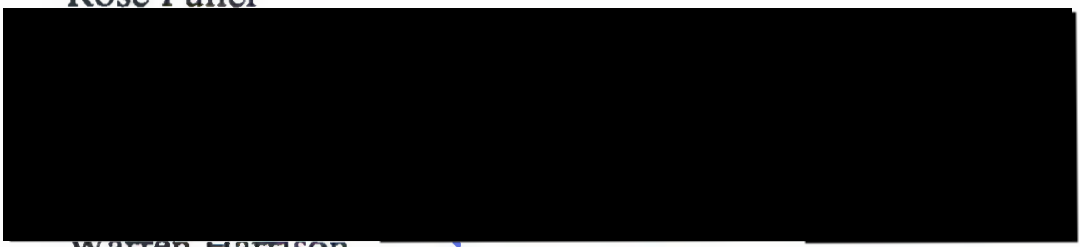

Wancentrantsoll

\section{APPROVED:}

Korr Schauman, Chair, Department or Electrical Engineering

C. William Savery, Interim Vice Provost forcGraduate Studies and Research 
Trust in the Lord with all your heart

and lean not on your own understanding;

in all your ways acknowledge him,

and he will make your paths straight.

Proverbs 3:5-6 


\section{ACKNOWLEDGEMENTS}

I would like to express my gratitude to Sue and Dick Naven whose constant encouragement and contribution have helped me finish this work.

I also want to thank Ms. Rose Fuller for all the constructive comments, and suggestions provided during the last part of this thesis. 


\section{TABLE OF CONTENTS}

PAGE

ACKNOWLEDGEMENTS..................................................................................

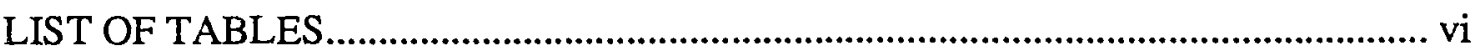

LIST OF FIGURES............................................................................................... vii

CHAPTER

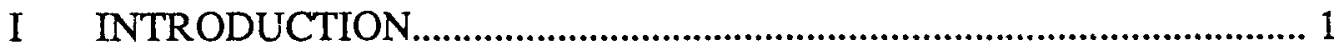

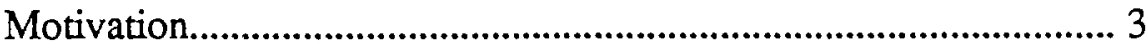

Overview of the System................................................................. 5

II BIOPHYSICAL PHENOMENA AND ELECTROPHYSICAL

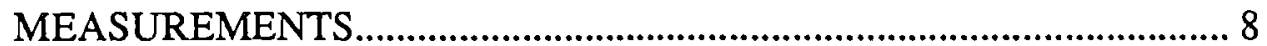

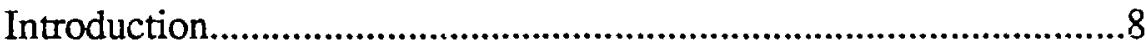

Methods of Monitoring and Predicting Ovulation............................ 9

Periodic Abstinence Method............................................................. 17

III CURRENT OVULATION MONITOR DEVICES.................................. 23

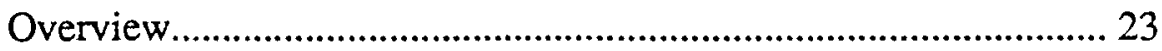

IV CURRENT METHODS AND THE OVULO-COMPUTER.................... 28

Suggested Parameters to Be Measured.............................................. 28

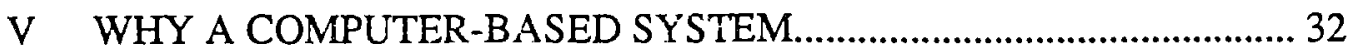

VI MEDICAL DECISION-SUPPORT SYSTEMS..................................... 35

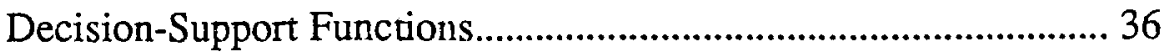

Knowledge-based Medical Systems............................................... 39 
VII THE OC DATA ACQUISITION SYSTEM............................................. 43

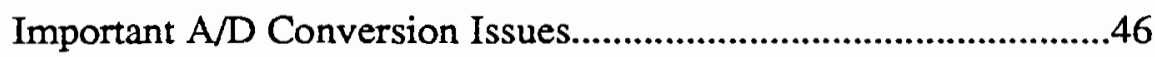

The A/D Converter Used in the OC............................................... 48

VIII THE OVULO-COMPUTER SYSTEM............................................... 50

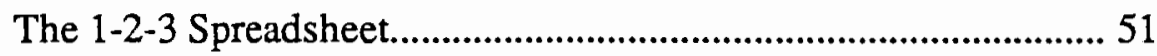

Implemented Functions................................................................ 52

IX DECISIONS AND RULE IMPLEMENTATION................................ 61

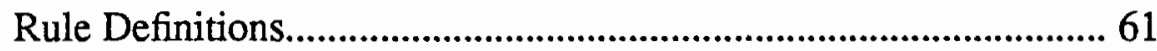

X PARTIAL EVALUATION OF THE OVULO-COMPUTER.................. 70

XI METHODOLOGY FOR EVALUATION OF THE OC.......................... 75

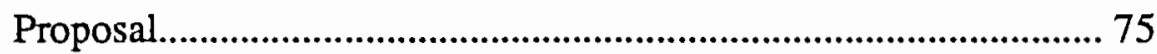

XII CONCLUSIONS AND FUTURE RESEARCH................................. 82

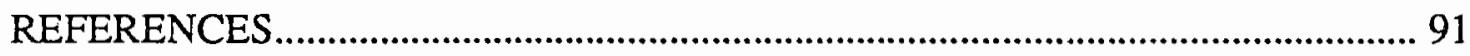

GLOSSARY 


\section{LIST OF FIGURES}

1. Probability of Conception vs. the Time of Intercourse Relative to Ovulation in the Human............................................................... 2

2. Block Diagram of the OC Data Acquisition System....................................... 44

3. OC's Menus Containing All the Functions Implemented.............................. 54

4. Example of Some of the OC Graphic Features......................................... 57

5. Critical Values as Considered by WHO's Rule...........................................62

6. Critical Values as Considered by Vermesh's Rule.......................................63

7. Example of the Critical Values as Considered by the Basic STR.............. 64

8. Example of the Critical Values as considered by the Temperature-Only Rule.

9. Average Line and Estimated Day of Ovulation as Determined by the OC-Albrecht Rule 66

10. Typical VER Values Around the Day of Ovulation. The Black Bars Show the Timing of the LH Peak............................................ 66

11. SER Values Around the Day of Ovulation. The Black Bars Show the Day of Ovulation as Estimated by the LH Analysis 68 


\section{LIST OF TABLES}

I Testing Results from Eight BBT Rules Applied to the Same

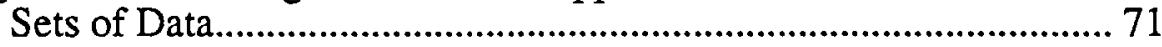




\section{CHAPTER I}

\section{INTRODUCTION}

Simple and reliable methods for predicting and confirming ovulation have been long desired by physicians and infertility patients. The main goals of such methods seek to provide great accuracy in the management of infertility as well as in the reliability of birth control methods.

Various health and research groups are searching for new ways to improve current methods of prediction and determination of ovulation and to develop new ones. Natural family planning programs search to improve the reliability of methods for defining the fertile period of the menstrual cycle and to extend their utility to periods of lactation, onset of menarche, and the approach of menopause. Artificial insemination and in vitro fertilization programs look for more complete information on the timing of ovulation and the period of optimum fertility to maximize the chances of conception while minimizing the cost and inconvenience of preliminary testing [4].

Ovulation itself cannot be observed externally. The only definite proof of its occurrence is establishment of pregnancy, which is normally the outcome being regulated. Therefore, it becomes necessary to predict the timing of ovulation on the basis of indirect physiological indicators which vary consistently with the time of ovulation [4].

As shown in Figure 1, the chances of conception from an act of intercourse are very low 6 days before ovulation, increase to a maximum during the day before ovulation, and fall to zero within 24 hours after ovulation [1]. 


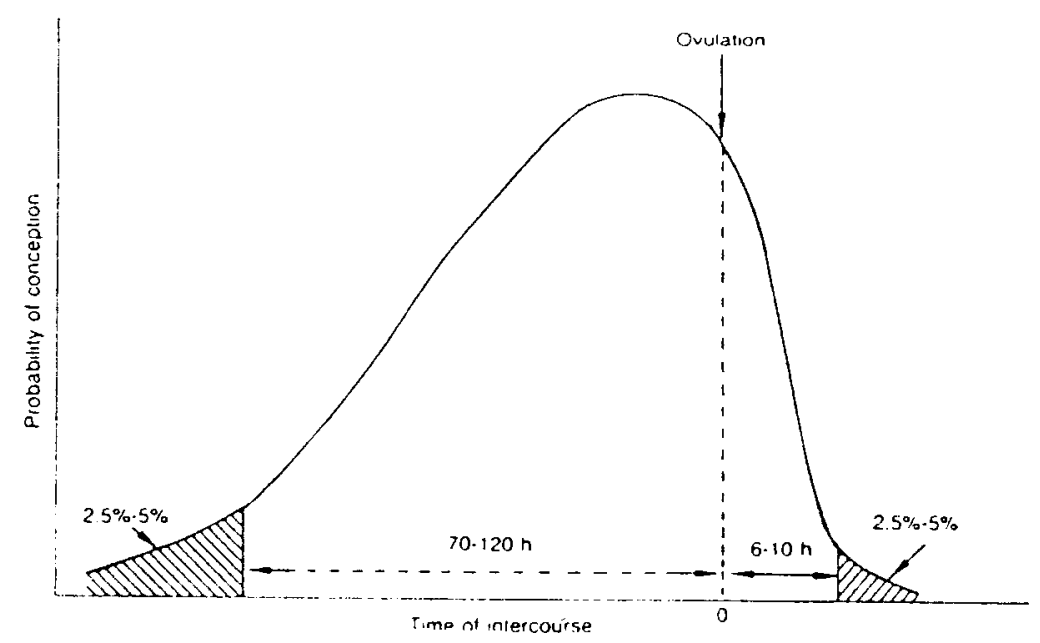

Figure 1. Probability of conception vs. the time of intercourse relative to ovulation in the human [1].

The scientific challenge then is to devise methods for identifying these periods of fertility and infertility with absolute certainty so that couples who use natural family planning have the same reliability as those using other modern family planning methods[1]. Brown et. al, states that the main difficulty in natural family planning for motivated couples is not the periodic abstinence required, which may in fact enrich the union. Rather, it is uncertainty in defining the fertile phase accurately, the excessive abstinence that is therefore required for maintaining adequate safety margins, and the loss of confidence resulting from unexplained pregnancies [1].

For decades it has been relatively easy to make the retrospective diagnosis of ovulation and even determine its approximate occurrence time. The events preceding ovulation are not always easy to monitor symptomatically and are extremely variable among women [5].

The prospective diagnosis of ovulation, however, is a problem that currently is without satisfactory solution. According to Kenigsberg, no method can achieve $100 \%$ confidence of prospective ovulation within 24 hours in the natural ovulation cycle. He 
adds, however, that the combination of some of the current reliable techniques will increase considerably the accuracy of new fertility monitors [5].

There have been many attempts to solve these needs. For over two decades, scientists have increased the research in this field. Important studies made in this field are discussed in the next chapter. Chapter 3 describes some home-use kits that have been proposed, some for detection only, and others for both detection and prediction of the ovulation timing. Also, more sophisticated devices, such as the ones based on ultrasound or hormone-based techniques, have been considered for years. Each of these devices has been based strictly on measuring only one parameter to make conclusions about what the ovulation conditions are. However, few satisfactory results have been obtained so far, and there are still several unsolved issues concerning accuracy, reliability, comfort, and cost.

\section{MOTIVATION}

The OVULO-COMPUTER concept emerged in response to several motivations in the field of fertility assessment.

\section{A Renewed Interest in Natural Family Planning}

More and more women are rejecting artificial methods of family planning and looking for reliable and easy-to-use natural methods. Artificial methods are not accepted by couples some because of ethical, religious, philosophical, health, and esthetic reasons. Therefore, methods to determine the fertile and infertile phases of the menstrual cycle can be used by couples as a contraceptive aid. They may appeal to people who wish to practice natural family planning, which implies abstinence from sexual intercourse during the fertile days of the menstrual cycle, or who wish to limit the use of barrier methods or coitus interruptions to the days of the menstrual cycle during which sexual intercourse 
can result in pregnancy [6].

\section{Safety Concerns and Side Effects of Contraceptives}

Closely related to the first motivation, extensive studies have shown that contraceptive methods, though so far more reliable than NFP methods, present many side effects that have become determinant for the couple when selecting which birth control method they decide to use. Side effects can range from mild to potentially life threatening. Some of the well known are: cordiovascular complications, pelvic inflammatory diseases, vaginal infections, and infertility. All these drawbacks encourage family planning programs to look for better methods that may lead to disregard of contraceptives or at least to reduce the use of them [7].

\section{Artificial Insemination and Special Treatments in Infertile Couples}

For several years, scientists have been doing research in an attempt to find the causes of infertility in couples. Their goal has been to develop special techniques to find the period of optimum fertility for conception. The success of some therapeutic measures, such as artificial insemination, depends upon adequate documentation and timing of ovulation. When pregnancy is desired by both fertile and sub-fertile couples, a fertility indicator can be used for timing intercourse to maximize the chance of conception, as well as for monitoring the administration and effects of therapy in infertile couples.

\section{Quality Patient Care and Traditional Methods}

Knowledge of a patient's past medical history is a critical factor in the delivery of quality patient care. In the event that patient information is unavailable, insufficient, or inaccurate at the time of assessment, as is frequently the case, the next line of inquiry is the patient's medical record. The traditional paper-based charting system used in record- 
ing menstrual cycle data has inherent limitations. The physical chart may be unavailable due to temporary mislocation or possession by other users who are reviewing or recording data. Even when the chart is available, poor organization, illegible handwriting and sheer volume may make retrieval of clinically significant information time-consuming, laborious, and incomplete [8].

\section{$\underline{\text { Need of Tools for Comparison and Evaluation }}$}

Tools to compare and evaluate methods for the prediction and detection of the ovulation timing have been desired for decades. Diversity in the interpretation of rules has been of great concern to many researchers [9]. Most physicians agree that the best reference tool to confirm ovulation is the LH serum technique. The sustained rise in the woman's basal body temperature (BBT) is a well documented confirmation of ovulation. Evaluation of these rules has frequently resulted in contradiction among research results $[9,10,11]$. Therefore, the implementation of a tool such as a computer based system for objective and reliable evaluations would be greatly appreciated by gynecologists, researchers, and practitioners in the field of fertility. This tool may also have a great impact in deciding which family planning method is more reliable and accurate.

\section{OVERVIEW OF THE SYSTEM}

In response to such a great need for a comprehensive, accurate, flexible, and reliable device for the prediction and detection of ovulation in women, a computerized system for measuring, visualizing, and interpreting relevant changes in the woman's body is proposed in this thesis. The main goals of this thesis are:

- Design a computer-based system for evaluation and comparison of rules concerning the ovulation in women. 
- Develop a more comprehensive fertility monitor based on more than one parameter.

- Eliminate some sources of human errors by automating some procedures in the collection and interpretation of data.

The major features of this system consist of acquiring, measuring, and interpreting different types of bio-physical variables involved in women's menstrual cycles, especially around the ovulation day. By integrating hardware and software technologies, this system automates as much as possible the tedious tasks of data gathering and interpretation. Decision making is enhanced by providing accurate data about the ovulation status; however, physicians, nurses, or practitioners will evaluate the most prudent course of action.

As mentioned before, methods of gathering and recording data concerning ovulation present limitations. By interfacing the sensors directly to the electronic monitoring system, the OC automatically records the patient data, eliminating the burden and errors of manual procedures. The system acquires real-time patient data from a number of biophysical electrodes. This system is also able to accept data manually from the user (e.g. symptoms), from any other physiological monitoring equipment, or from other medical departments, such as ultrasound or assay laboratories.

In order to facilitate the review, comparison, evaluation, and interpretation of the data, the $O C$ provides the following features.

- Graphics: Graphical trends are provided on either a video display or a dot-matrix or laser printer.

- Charts: A summary chart displays the results after the rules have been applied to the data. The table-like features of the 1-2-3 spreadsheet allow the user to visualize numerical and text variables on the screen or at the printer.

- Decisions: Based on the most generally accepted rules, the OC evaluates each of 
rules to a specific set of data and provides the user a diagnosis of the fertility status. Some of the rules provide also a probability of the results calculated.

All of these features enhance the usefulness of the system and allow the user to draw more accurate conclusions from absolute values, trends, or inferred values from combinations of many parameters.

The OC could be considered as a research support tool in the prediction and detection of ovulation, and for comparison and evaluation of rules to determine the fertile and infertile phases of the woman's menstrual cycle. Although this system has been designed exclusively for clinic and hospital use, there is also a future goal to build a simple, easyto-use device based on the results of this system for the consumer. 


\section{CHAPTER II}

\section{BIOPHYSICAL PHENOMENA AND ELECTROPHYSICAL MEASUREMENTS}

\section{INTRODUCTION}

\section{Overview on the Menstrual Cycle}

Unlike male fertility, which is relatively constant, female fertility is cyclic; ovulation normally occurs once a month and is regulated by a hormone system that involves the hypothalamus, pituitary gland, and ovaries. Understanding how these hormone patterns affect the maturation and release of an egg from the ovary, and uterine preparation for implantation, is fundamental to understanding how natural family planning methods work [12].

The menstrual cycle needs to be distinguished from the menstrual period. A woman's menstrual cycle begins with the first day of her menstrual flow and last through the day before before the next menstrual flow starts. The menstrual period is the time of menstrual flow, usually lasting 3 to 7 days [12].

Cycle phases are traditionally named either by the changes that occur in the ovary (the follicular phase and the luteal phase) or by the changes that occur in the lining of the uterus (the menstrual phase, the proliferative phase, and the secretory phase). The first day of menstrual flow, by convention, is called day 1 of the menstrual cycle. The time interval between ovulation and the onset of menstruation is consistently close to 14 days for all women (normal range 12 to 17 days) [12]. 
A woman is most likely to conceive if fresh sperm are present in the reproductive tract when ovulation occurs. The woman's oocyte retains maximal potential for fertilization for 12 to 24 hours. Sperm retain potential for fertility for a significantly longer interval, 100 hours or more. Intercourse during the 4 days preceding ovulation results in the highest conception rates during a menstrual cycle [12].

\section{Charting}

Menstrual cycle charting is based on observations of the physiologic changes during the cycle. The actual charting carries the observation one step further, to precise documentation of the changes. Charting is a method for planning or preventing pregnancies by identifying the fertile days of a woman's menstrual cycle. It is not, therefore, a method of contraception. Instead, the user must rely on abstinence or other contraceptives during her fertile days. Moreover, many women rely on charting methods as a means for conceiving [12].

When charting is combined with other methods of family planning during the fertile period, it is called fertility awareness. When charting is used for birth control in combination with abstinence during the fertile period, it is called natural family planning, and includes the rhythm or the calendar method, the basal body temperature method, the ovulation or Billings method, and the sympto-thermal method [12].

\section{METHODS OF MONITORING AND PREDICTING OVULATION}

In this section an overview of some of the most recent and well accepted methods will be given. Special emphasis has been given to selected methods based on their relevance as well as on the parameters that can be electrically measured and adapted to a computer system. 
Electrical Resistance Method

Salivary and cervicovaginal changes in electrolyte content through the menstrual cycle have been described for decades $[13,14,15]$. During the last few years, several studies have been made in an effort to understand these changes. The most significant work on this technique was done by Albrecht et. al in 1985 [16]. Since then, many other investigators have evaluated and proved the importance of this work. Albrecht found that mean changes in the Vaginal Electrical Resistance (VER) indicate a clearly defined nadir on the day of the LH peak. The VER mean at LH peak day was significantly lower than the overall mean for all other days. Thus, the absolute value of VER, combined with the rapid decline in the last three days before this nadir, are interpreted as a sign of imminent ovulation. The most significant change in VER was on the day after the LH peak, when the mean VER was $70 \%$ higher than on the previous day. This change coincided with the day of ovulation, which in most cases, is assumed to occur within 24 hours of the LH peak [16].

In this same study, Albrecht found that changes in the salivary electrical resistance (SER) also followed a predictable pattern during the preovulatory phase. A peak SER value was consistently seen 5 to 6 days before the cycle day of the LH surge. After the SER peak, SER values remain low for 3 days and increase again before ovulation. This SER peak, therefore, could be used to predict the day of ovulation.

The physiological causes of these changes have also been explained. The changes in VER, Albrecht states, are primarily caused by changes in the ionic content of the cervical mucus and its volume. SER changes which are consistent with changes in sodium concentration are thought to be related to the effect of estrogen on the renin-angiotensin system [16]. The physiologic basis for the nadir in VER during the periovulatory days is justified because of the well known properties of cervical and vaginal mucus secretion during those days. The increase in volume and ferning of the mucus are at a maximum 
coincident with the preovulatory estrogen peak. Ferning is due to $\mathrm{NaCl}$ crystal formation in the presence of mucin; the 10- to 12-fold increase in volume of that mucus should provide a high conductance and therefore, a low electrical resistance [15].

Most of these studies have confirmed the usefulness and consistency of these salivary and vaginal changes $[5,11,16,17,18]$. However, because these methods were developed recently, more studies are needed to establish the reliability for some cases, such as in women with variable cycle lengths, or when abnormal vaginal secretions or infections are presented [15]. Some studies have questioned the usefulness of VER and SER techniques $[19,20]$. However, the differences in the results obtained were probably due to differences in the device calibration and the product used (The Peak Monitor).

SER and VER method has also proven to be reliable when used in cycles where clomiphene citrate was used to induce ovulation. A protocol for timing inseminations developed by Fernando et. al showed that inseminations based on the SER peak would have been timed within 24 hours of the thermal nadir in 14 of 15 cycles [18]. In another study, Fazleabas adds that combining the prediction capability of the SER peak with the more precise indication of ovulation provided by the VER trend could result in accurate timing of the inseminations. Thus the convenience of advance scheduling is made without sacrificing accuracy. Also, this method would avoid performing multiple inseminations as required by other methods.

\section{Tests Based on Hormone Assays}

Useful hormonal determinations include daily assays of serum or urinary LH, urinary estrogens (or estrogen metabolites) or serum estradiol, and serum progesterone or urinary pregnanediol [11].

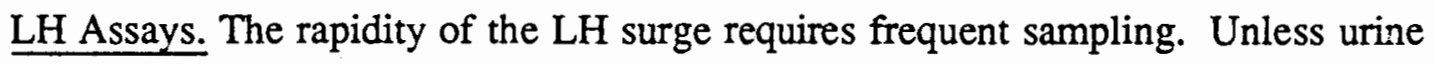
is used, this presently involves repeated venipuncture. Still, it often cannot be used 
effectively for frequent serial sampling because of problems with trauma, infection, and red cell depletion [4].

Radioimmunoassay (RIA) of LH is now readily available in most hospitals and through commercial laboratories. Daily assay of serum LH in midcycle can detect the LH surge, which is presumed to occur before actual ovulation. The temporal relationship of the LH surge and ovulation has been studied by several investigators [11]. According to Campbell, assays for progestogens in serum, saliva, and urine meet high standards with respect to accuracy, bias, and sensitivity, as do assays for estrogens in serum [4].

Estrogen Assays. Serum estradiol demonstrates a characteristic peak approximately 1 day before the LH surge and 37 hours before ovulation. Serial determinations of serum E2 at midcycle, thus, can detect the time of ovulation with a fair amount of accuracy. Rapid RIAs for serum E2 are available but require a specialized laboratory and are timeconsuming and expensive. Urinary estrogen assays have been applied for detection and timing of ovulation. The preovulatory peak of urinary estrogens ranges between 40 and 100 micrograms $/ 24$ hours. The mean interval between the first observable rise and the estrogen peak is 5.5 days. The day after the estrogen peak (day of the LH surge) is believed to be the day of maximal fertility [11].

Urinary LH Analysis. Urine collection is considered to be easier than serum collection, however, it may be socially unacceptable in some cultures. The problem can be exacerbated by any requirements to accumulate and store urine specimens either for 24 hour periods or as serial samples. Use of short-term, spot, or early morning urines will help. The best approach may be the use of self-tests that do not require supervision or storage and thereby minimize any embarrassment or inconvenience [4].

In a recent study, Kenigsberg reported that monoclonal antibody technology along with enzyme-linked immunosorbent assay (ELISA) methods have renewed interest in urinary hormone assays where both the body fluid and technical skills are readily 
available to the patient [5]. The basic technology used in these kits involves combined monoclonal antibodies to LH coupled with a second antibody reaction resulting in an enzyme activation producing a color reaction (usually blue). Depending on the amount of reagents, the reaction can be triggered at a certain level of LH (usually $40 \mathrm{mIU}$ and 20 $\mathrm{mIU}$ ) to give an "all or none" and an "in between" response, respectively, readable by the naked eye. Kenigsberg added that with simultaneous collections of serum and urine, correlation between radioimmunoassay (RIA) results in serum and ELISA results in urine is virtually $100 \%$ [5].

\section{Clinical Potentials of the Cervical Mucus Constituents}

Cervical mucus functions have not been totally explained [21]. It presents, except at midcycle, a mechanical barrier to particulate (including sperm) that might otherwise reach the uterine cavity. A major function of cervical mucus is antimicrobial activity which is accomplished by the presence of leukocytes, which are decreased at midcycle, and by other inhibiting substances which are not decreased at midcycle and effect microbial inhibition throughout the cycle [21].

The $\mathrm{pH}$ of the cervical mucus and endocervical canal is important in relation to fertility, since sperm motility is reduced when the $\mathrm{pH}$ falls below 6.5. Infertile patients are more likely to conceive if the $\mathrm{pH}$ is alkaline, and changes in the $\mathrm{pH}$ have been noted in women taking an oral contraceptive [22]. Spermatozoa are highly susceptible to changes in the $\mathrm{pH}$ cervical mucus and maintain their activity best in a $\mathrm{pH}$ of 7 to 8.5 . This is the $\mathrm{pH}$ range of normal midcycle cervical mucus [23]. There are, however, conflicting reports in the literature about the normal range for endocervical $\mathrm{pH}$ and whether the degree of acidity or alkalinity is under ovarian control [22]. According to FordneySettlage, similar problems exist for the glucose fertility tester and indicine tests. He adds that the chief problem with these techniques is the lack of regular predictable changes 
early enough prior to ovulation [21].

\section{Ultrasound Method}

Ultrasound has been used extensively during the past decade to monitor follicular growth and to time ovulation in spontaneous as well as stimulated cycles [17]. Follicles are imaged as areas having ultrasound density identical to the adjacent full bladder but contrasting with surrounding, less dense ovarian tissue. Objects of 5-10 mm are clearly discernible to $\pm 1 \mathrm{~mm}$. Ultrasound is capable of detecting growing follicles as early as the first 5 days of the menstrual cycle. It can register the presence of pre-ovulatory follicles of at least $15 \mathrm{~mm}$ diameter at 2-3 days prior to ovulation, and can sometimes indicate the presence of a detached, ripe ovum/cumulus complex within a Graafian follicle approximately 16-24 hours prior to ovulation. Although ultrasound serves as an excellent index of imminent ovulation, several reports indicate that there is a rather wide range of follicular sizes $(17-27 \mathrm{~mm})$ from which mature, fertilizable eggs may be aspirated. This variability in attained size and attendant steroid content limits the predictive precision of ultrasound. However, since it can detect the collapse of the antrum following ovulation, ultrasound has an internal retrospective check on the accuracy of its own predictions [4].

The advantage of this method is that it is noninvasive and the evidence it provides is persuasive. It does require frequent clinic visits and a large investment in equipment, and thus it is expensive. Nevertheless, its documentation of ovulation is most reliable in retrospect, that is, when follicle collapse or a reduction in follicle size is seen to have occurred. For these reasons, this method can not be used for routine ovulation prediction [17].

According to Campbell, ultrasound must be done serially if accurate information is to be obtained. He adds that, until the beginning of the fertile period, even serial ultrasonic scans cannot distinguish which of the remaining 4-6 growing follicles will be 
atretic and which will eventually ovulate [4]. In a more recent study, however, Vermesh et. al [2] demonstrated that ovulation was detected by transvaginal ultrasound in all cycles, which renders this method an excellent tool for positive confirmation of ovulation. He adds that the advantage of ultrasound over other methods used for retrospective detection of ovulation (i.e., serum $\mathrm{P}$ and BBT) is that it can pinpoint the day of ovulation and not merely the fact that ovulation has occurred. In addition, serial ultrasound examinations provide valuable information about the progressive growth and development of the follicle(s). In this study, transvaginal ultrasound was used. This method has been shown to have a greater resolution than the classical transvesical route of scanning, especially when patients are obese or have pelvic adhesions [2].

\section{$\underline{\text { Basal Body Temperature (BBT) }}$}

It has been noted for more than 80 years that there are body temperature changes throughout the menstrual cycle. The charting of daily thermometer readings has been practiced for more than 40 years [5]. BBT charting has long been a valuable tool for detecting the postovulatory phase of the menstrual cycle and as a simple aid for diagnosing ovulation in women who have had difficulty conceiving. The clinical usefulness of charts produced by women monitoring their BBT has been limited, for the most part, by fragile mercury thermometers requiring three to five minutes to register an accurate temperature, and by the inaccuracy of the charting process when left to the patient. The temperature may be read or recorded incorrectly, or forgotten, or may be taken at times other than before rising in the morning. Also, among physicians there exists some sense of frustration over the lack of usefulness and interpretation of the BBT graph. One of the reasons is the lack of any generally accepted criteria for objectively interpreting BBT graphs [24].

The term "basal" means the body temperature at rest, unaffected by any activity. It 
is called the "waking temperature", because it is taken immediately upon waking from rest.

A fertile woman who takes her waking temperature daily under the same conditions will notice a biphasic pattern during her cycle. "Biphasic" means it has two levels. First, a lower level from menstruation until about the time of ovulation; then, a rise to a higher level where it stays until the end of the cycle. Ovulation occurs within a few days before or after the rise to an overall higher level. The temperature rise indicates when the postovulatory infertile time is beginning and confirms that the bleeding 1-2 weeks later is really menstruation. Women are able to know if pregnancy has occurred once the temperature is high for $18-20$ days [12].

$\mathrm{BBT}$ rises $0.2-0.5^{\circ} \mathrm{C}\left(0.4-1.0^{\circ} \mathrm{F}\right)$ due to the midcycle increase in progesterone caused by the transformation of the ovarian follicle to the corpus luteum in response to the LH surge. Although progesterone begins to rise about 24 hours after the beginning of the LH surge or 8 hours before ovulation, the rise from the hypothermic plane existing during the follicular phase to the hyperthermic plane present during the luteal phase can be observed only an average of 1 day after ovulation. Moreover, since temperature shifts also occur in response to other agents, the menstrual cycle shift may be verified by a continuation of the elevated temperatures for at least 3 days. Therefore, being strictly retrospective, BBT cannot predict ovulation: It can only detect probable ovulation and can only predict the end of the fertile period [4].

BBT charts could serve as a "low-tech", inexpensive, and reliable way for documenting ovulatory cycles once they have occurred. They roughly show 10-14 day luteal phases in women with cycles with variable lengths. Used in the context of a fertile couple attempting pregnancy or even for timing at donor insemination, they are useful adjuncts. Because of the intrinsic variability of cycle-to-cycle oocyte release and the confounding factors affecting measurement (illness, activity, drugs), however, the BBT is 
not adequate for precise, prospective ovulation prediction [5].

\section{Sialic Acid Method}

Sialic acid ( $\mathrm{N}$-acetylneuraminic acid) is a normal constituent of body mucins. According to Calamera, sialic acid in cervical mucus shows cyclic variations [25]. The cervical mucus shares many common properties with other body mucins. Sialic acid content in cervical mucus decreases during the follicular phase preceding ovulation, and it increases significantly during the luteal phase and pregnancy. Salivary sialic acid shows a higher concentration during the follicular phase until 4 or 5 days before the LH surge, when a peak of maximal activity is observed. Then, there is a progressive diminution towards the time of ovulation, followed by a less evident increase. In this study, Calamera concluded that their results indicated that saliva sialic acid determinations are easy and inexpensive means of studying the menstrual cycle [25]. So far, no commercial device has been developed based on this technique.

\section{PERIODIC ABSTINENCE METHOD}

Sexual abstinence is considered to be the safest method of family planning, although not the most practical one. There are some factors that interfere with the effectiveness and practicality of this approach to family planning. First and foremost is irregularity of the menstrual cycle among women and even in the same woman over an extended period of time. If all women had regular cycles, calendar rhythm would be a reliable contraceptive method. Occasional anovulatory cycles, however, occur even in women who are known to have regular cycles and are seen with greater frequency in postpubertal and perimenopausal women [11].

The effectiveness of periodic abstinence depends largely on the strength of a couple's motivation to avoid pregnancy and the woman's ability to interpret signs and 
symptoms of the fertile period. A major problem has been the unwillingness of one partner or the other, usually the male, to agree to long periods of abstinence. Another problem is that women sometimes have difficulty in identifying their fertile periods, especially if they are breast-feeding, have vaginal or other infections, or are approaching menopause [26].

Moghissi concluded that a simple, objective, self-administered technique of ovulation prediction, such as a kit for home use will undoubtedly improve the efficacy and acceptability of periodic abstinence as a method of family planning [11].

Among many possible techniques of natural family planning, the following three have been subjected to clinical trials and deserve emphasis.

\section{Calendar Method (Ogino-Knaus).}

This method identifies the time of ovulation from data recorded over the previous 6 to 12 months to determine the intervals between menses. The range of possible ovulation dates and an additional 3 to 4 days before and after these dates dictate the period of sexual abstinence. Although the length of the normal luteal phase is believed to be relatively constant ( $14 \pm 2$ days), irregularities of the menstrual cycle are a frequent source of error, and the method has been associated with failure rates ranging from 14.4 to 47 pregnancies/100 woman-years [11]. A recent device called L-Sophia bases its principles of calculation of the periodic abstinence on this method and BBT [27].

\section{Cervical Mucus (Ovulation) Method}

This method relies upon a sign, the discharge of cervical mucus, which is essential to human fertility. The menstrual cycle begins with the first day of menstruation and ends the day before the beginning of the next menstruation. The menstrual flow lasts from 3 to 7 days. Then, five phases of the cervical mucus pattern are recognized: phase 1, 
dry days; phase 2, early preovulatory days; phase 3, wet days; phase 4, postovulatory days; and phase 5, late postovulatory phase [11].

The fertile, or unsafe, period is presumed to start on the 1st day in which postmenstrual mucus is observed (phase 2) and to continue until the fourth day after the clear lubricative cervical mucus (peak day) appears, a period that can last from 7 to 14 days. All subsequent days are considered infertile and safe for intercourse. A period of formal indoctrination is believed to be essential for the successful practice of this technique [11]. A more detailed explanation to this method is found in Appendix A. Billings et. al and Weissman et. al have claimed a high degree of effectiveness in well-motivated couples who have been adequately trained [11].

\section{Sympto-Thermic Method (STM)}

This method employs not only observation of cervical mucus but also a calendar calculation during the preovulatory phase and the BBT shift during the postovulatory phase of the menstrual cycle to determine the time of fertility. The calendar calculation (21 is subtracted from the shortest cycle length in the preceding 6 months) establishes the last day on which sexual relations are permitted during the preovulatory phase. Abstinence begins before this day if mucus in noted. Abstinence continues until the third day of the BBT rise. After that day, sexual relations may be resumed until the onset of menses [11].

According to Moghissi, this method is probably the most reliable method of periodic abstinence. Provided that the couples are well-motivated, disciplined, and follow the technique closely, a pregnancy rate of less than $1 / 100$ woman-years (Pearl index) as reported by Roetzer may be expected. In randomized prospective studies a pregnancy rate of $9.4 \%$ (Pearl index) was observed among STM users during the year following formal entry into the study [11]. 
There are several symptoms or body signs that reflect hormonal changes in the menstrual cycle [12]. These signs play an important role and it can be helpful to record them because they can provide confirmation to other more accurate signs (e.g. SER, VER). Most of these signs constitute the basis of the Sympto-Thermal Method. Some of these symptoms considered in the STM are:

Tissue-Paper Exam of the Cervical Mucus. An important sign observed in NFP is the cervical mucus symptom. Fertile women notice a cervical mucus discharge at the vaginal entrance during part of their cycle. This is a normal occurrence. Cervical mucus is important for fertility. The start of the mucus flow means that a follicle is maturing toward ovulation. The possibly fertile time of the cycle has begun.

In a typical cycle the mucus is at first sticky and cloudy, then progresses to a stretchy, clear, lubricative condition like raw egg-white, before changing back to sticky or dry about the time of the temperature rise. There may be dry days after menses and during the established high-temperature phase [12].

Vaginal Sensations. Another important examination is the awareness of vaginal sensations. What women feel through this attentive awareness is as important as what they see by doing the tissue exam. The three types of sensations are [12]:

Sensation-dry: may be a positively dry, somewhat disagreeable, slightly itchy feeling, or just a feeling of "nothing".

Moistness: a feeling of "something" as if secreting moisture or something flowing Down inside the vagina or small bubbles bursting inside the vagina.

Lubrication: a feeling of slipperiness at the vaginal entrance, or a very distinctly runnywet feeling, both due to highly-fertile egg-white mucus (EW-M).

For the purposes of interpretation sensation-dry, moistness and lubrication are equivalent to tissue-dry, sticky mucus and EW-M respectively. The peak day is the final day of EW-M; that is the final day on which any trait of EW-M is still noticed, namely 
clear, or stretchy an inch or more, or lubricative, causing the tissue to glide or a slipperywet feeling at the vaginal entrance [12].

Cervix Signs. Internal cervical exam have been found very helpful for identifying fertility. There are basically two observations:

- Noticing cervical mucus changes by touching the cervix.

- Noticing mucus at the cervical os (mouth of the cervix) by compressing the cervix.

The cervix typically changes according to the following cycle pattern: The cervix becomes firm, low, closed, and dry during the Relativy Infertile Time. Then, it becomes soft, high, open, and wet during the Possibly Fertile Time; and changes back to firm, low, closed, and dry during the Completely Infertile Time [12].

When compressing the cervix to identify the start of mucus at the cervix in the beginning of the cycle, two types of observations are identified in the test [12]:

Cervix-dry. This sign means no mucus is obtainable by compressing the cervix, or else there is at most only a continuous thick yellow or white mucus, which is not cervicai.

Cevix-Wet means cervical mucus is now present that will draw threads, whereas before there was none. During the highly fertile time typically "cascades" from the cervix.

Secondary Signs. Other useful signs for the detection of the days around the ovulation day are [12]:

Intermenstrual Pain is thought to be related to the fallopian tubes rising up around the ovaries near the time of ovulation. This sign is felt as a sharp pain or as a crampiness, somewhat like the onset of mild labor contractions. It is usually noticed only for a few hours, and rarely for more than two days. Intermenstrual pain, as defined here, seems to be associated with peaking estrogen [12].

Breast Tenderness may begin with a prickly, tingling sensation, sometimes a sharp pain in the breast, more toward the nipple; some women describe it as "having sore nipples". 
This may change into a heaviness deeper in the breast tissue and can be experienced as breast "tension" or "fullness", and finally as tender "lumpiness" just before menses. Breast tenderness reflects rising and elevated progesterone levels [12].

Intermenstrual Bleeding may be noticed around peak day. It is believed that most women have at least microscopic ovulation spotting, but only some that can be observed by the naked eye. Intermenstrual bleeding may be noticed as red, blown, pink or yellowish strands in the mucus, or as full bleeding around the time of ovulation, apparently due to a sudden drop in estrogen [12].

Bleeding other than menstrual period can be pathological bleeding, implantation spotting, breakthrough bleeding from excess or prolonged estrogen stimulation, or can occur whenever there are erratic fluctuations in the hormones upon which the uterine lining depends for its support. In particular, since the rebuilding of the uterine lining each cycle depends on estrogen, "withdrawal" bleeding can occur whenever there is a drop in estrogen (at ovulation time, for instance) [12].

Ordinarily, women can distinguish menstruation (menses) easily, however, sometimes there can be confusion. If there is doubt, those women who are charting temperature know that the only bleeding which is true menstruations is the bleeding that comes at the end of a high temperature phase. All bleeding other than true menstruation is considered fertile [12]. 
CHAPTER III

\section{CURRENT OVULATION MONITOR DEVICES}

\section{OVERVIEW}

\section{Fertility Monitors Based on SER and VER Methods}

CUE Fertility Monitor. The CUE Fertility Monitor (Zetek, Inc., Aurora, CO), is a battery-operated device with a digital readout that indicates the measured resistance at the two sensors one oral (saliva) and one vaginal (vaginal mucus). The measurement process requires about 10 seconds. A constant, alternating current source was used to minimize polarization effects at the electrodes. Calibration of the device is in arbitrary units which are directly proportional to resistance in ohms. A tenfold difference in VER and SER prevented a direct ohmic readout in the instrument because it was designed to be simple to use. The conductive electrodes of the sensors were stainless steel [16].

The results of this study indicate that SER may be used to obtain an indication of the beginning of the fertile phase of the cycle by predicting ovulation 5 to 6 days in advance, whereas VER enables a more short-term indication and later a confirmation of ovulation. Thus, the two methods may be combined to accurately define the fertile phase of the menstrual cycle. Albrecht concluded in his studies that this method is a simple but reliable tool for the management of infertility and an aid to the practice of NFP [16]. Later studies have confirmed the accuracy and usefulness of the CUE monitor for the prediction and detection of ovulation $[5,15,17,18]$. 
Urinary Hormone-based Kits

There have been several urinary LH kits developed during the last decade. These methods rely on a two-site immunospecific assay in which an enzyme is linked to an antibody specific for the beta subunit of $\mathrm{LH}$, which produces a blue color in the presence of substrate. The intensity of the color change is proportional to the amount of LH present. Results are obtained by detecting a consistent color change using as reference a surge guide provided by the kit. Daily sampling is necessary beginning on day 11 of the cycle until a sustained thermogenic shift in the BBT is identified [20].

In a recent study made by Rodriguez et. al [28], two immunoassay kits Ovustick (Monoclonal Antibodies, Inc., Mountain View, CA) and TestPack (Abbott Laboratories, Abbott Park, IL) were analyzed. These home-use kits are based on the detection of the day of the urinary LH surge. In this study, both devices predicted ovulation in $85 \%$ of the cases. TestPack provided faster results and was easier to use than the Ovustick. TestPack also had another advantage: a first morning urine sample, instead of a midday sample, could be utilized. Use of the first morning urine is more convenient for women who work outside the home. Because the results were similar with the two tests and use of the TestPack was more convenient and easy, Rodriguez suggests that of the two methods of detecting ovulation, TestPack appears to be better [28].

Other devices based on the principle of detection of urinary $\mathrm{LH}$ surge are: Discretest (Organon, Oss, The Netherlands), Right Moment (Seragen Diagnostics Inc., Indianapolis, IN), and First Response (Tambrands Inc., Palmer, MA). In a study in 1987, Vermesh et al [2] demonstrated that First Response predicted ovulation only in 53.3\% of the cycles, which makes this device an inaccurate predictor of ovulation. 


\section{Kits Based on Cervical Mucus Properties}

The OvuTest. This device known as "Determine; The OvuTest" (Ventco Medical Trading Company, Montreal, Quebec, Canada) was designed to determine the optimal time for human conception by detecting the presence of the protein, uteroglobin (UTG), in cervical mucus. The device is a vaginal probe, connected by an insulated electric wire to a metering unit powered by a 9-volt battery. In indicator needle on the metering unit deflects in the appropriate direction when optimal conditions for conception are present. The device is described as having the operating principle to measure the electrochemical charges resulting from the combined charges of proteins that bind differentially to the two metals of the probe's electrodes. This might reflect a variety of changes in fertilityassociated compounds. The manufacturer claims that the probe specifically detects the protein uteroglobin (UTG; or blastokinin), and that UTG is present in the vagina or cervical mucus only during the time of ovulation. However, Daniel et. al proved that results obtained with the OvuTest were "variable, indeterminant, and erratic, and therefore, useless for determining the time of ovulation" [29]. It was also shown in Daniel's studies that obtaining the meter reading takes at least 2 minutes, which adds more difficulties to this method.

\section{$\underline{\text { BBT-based Kits }}$}

The small increase in BBT has spawned a number of technical innovations that make its occurrence more obvious: mercury thermometers with expanded scales; electronic thermometers, some supplemented with the microcircuitry required for data storage and computation; and recording charts with vertically expanded scales [4]. Some of the most common devices based on the BBT technique are:

Bioself 110. The Bioself 110 device (Bioself Canada, Inc., Montreal, Quebec, Canada) is a hand-held, non-invasive that measures basal body temperature and cycle 
length, and automatically identifies the fertility and infertility phases of the menstrual cycle. This instrument measures BBT orally, vaginally, or rectally in 2 minutes and keeps track of the last 64 temperatures and the length of the last six menstrual cycles. A microprocessor with a mathematical algorithm identifies the upward shift in BBT that follows ovulation, and through the use of light signals, it provides the user with an indication of her level of fertility: a green light indicates "infertile", a flashing red light indicates "fertile", and a constant red light indicates a transitional stage during which the woman is "probably infertile". The memory of the Bioself 110 can be retrieved in a graphic form by inserting the device into a special printer, which was also provided by Bioself Canada, Inc. [30].

L Sophia. This device is based on the basal body temperature and the Ogino method. It records the daily temperature automatically and also some other symptoms, such as fever, bleeding, etc. The user is able to see a bar graph on a digital screen showing the temperature during the whole cycle. By flashing a series of figures, L Sophia indicates the fertility status.

According to the reference manual, to use L Sophia for birth control, it is absolutely necessary to adhere to the following measures, because it is not possible to obtain reliable data if you fail to observe even one of the measures; and without this reliable data, you cannot carry out birth control securely [27].

- Measure the temperature each morning at the time of awakening, while in bed, before rising. (Do not rise, do not move about.)

- Do the measuring at the same time daily.

- Measure the temperature daily.

- When the user has a cold or other illness with fever, do not record the temperature.

Since this is a recent device, no evaluation reports have been published so far. 
Serum Hormone Assays Products

These are probable the most reliable and accurate devices currently developed for the detection of the day of ovulation. However, they present the inconveniences, such as the patient has to make visits to the clinic during many days, and also requires blood tests. Examples of some of the companies that manufacture these type of products are: (LH, FSH, and P) (Farmos Helsinki, Finland), (LH-polyclonal, P) (Diagnostic Products, Los Angeles, CA), (LH) (Hi-Gonovis, Mochida Pharmaceutical Co., Tokio, Japan), (LH) (Serono Diagnostics, Randolph, MA). 


\section{CHAPTER IV}

\section{CURRENT METHODS AND THE OVULO-COMPUTER}

There is little doubt of the usefulness of fertility monitors. Both physicians and infertility patients are demanding simple and reliable devices for monitoring ovulation. It has been shown that the goals of the methods mentioned in the previous chapter have been similar: to provide an accurate and easy-to-use device that may help doctors and/or patients keep track of ovulation timing. It has been evident, however, that not all of these methods have been successful. Later studies which have attempted to evaluate and confirm the usefulness of any particular method have shown that few of these methods fulfill $100 \%$ of what they claim, and some of them have even been shown to be contradictory or highly inaccurate.

On the other hand, even the most current successful ovulation devices do not provide an efficient way to keep track of past medical history. As mentioned before, some of these kits detect the changes in the ovulation timing by turning lights on and off, by changing colors of substances, by the distance a needle indicator deflects or by using arbitrary units. That shows that there is a lack of standards of what to measure and how to measure it.

\section{SUGGESTED PARAMETERS TO BE MEASURED}

The purpose of the $\mathrm{OC}$ is to integrate a series of useful variables in the prediction and detection of ovulation. Because there is no absolute rule as to what should be measured or how many parameters should be used, the OC system should be flexible enough 
to enable the user to add new variables to the system whenever possible. Furthermore, it has been reported in several studies that in order to reach higher accuracy in predicting and detecting the timing of ovulation, it is necessary to combine two or more methods [5, $11]$.

Keeping track of ovulation timing using methods based on ultrasound or hormone assays presents some problems. First, these tests require very specialized equipment and therefore, are too expensive. Second, none of these methods predict the day of ovulation far enough in advance to be of use as a contraceptive method. Third, it has been reported that some of these methods cause discomfort for the patients or take a relatively long time, i.e., 5 minutes for ultrasound $[2,6,11]$. Fourth, as reported by Fehring, to achieve rates close to $100 \%$ certainty in detecting ovulation with some methods, such as urine LH self test kits, testing should be done twice a day, which is inconvenient, expensive, and time consuming. For these reasons, ultrasound and hormone assay methods are not considered as potential techniques in our system.

Methods based on physical or chemical properties of the cervical mucus have been reported to be not very promising $[21,29]$. Techniques based on physical properties are to a certain degree successful; however, they have some inconveniences, for example, how to extract the cervical mucus, discomfort for the patients, and more importantly, reliability. Considering the chemical properties in the cervical mucus, although many parameters change in response to the estrogenic and the ovulatory phase of the cycle, few change early enough or in a consistently great enough manner to be useful for ovulatory prediction in time to avoid conception. Four days before ovulation is the minimum time for sperm in the female reproductive tract to undergo death. To date, no unique constituent of cervical mucus present as early as that has been identified which could be simply and easily determined. Most properties require at least a direct sampling method and a simple laboratory as well [21]. Nevertheless, the analysis of some of these chemical 
parameters could be considered in our system in the future, for the purpose of encouraging the research on the ionic composition not only of cervical mucus, but also of other body liquids, such as urine or saliva.

After analyzing the most recent studies on ovulation monitoring, it has become clear that one of the most promising techniques developed so far is the Salivary and Vaginal Electrical Resistance Method. This technique has some clear advantages over other methods. First, it was shown to be highly reliable. Second, the changes observed in electrical resistivity are significant; thus, it does not require extremely sensitive electrodes, and this makes the interpretation of the data easier. Third, the procedure to make the measurements is relatively easy and only takes about 10 seconds. Fourth, and most important, this is the only method that so far has been demonstrated to provide ovulation prediction early enough ( $>5$ days) to be used in natural family planning as a method of birth control. It is for these reasons that SER and VER are the most important parameters to be measured in our system.

The Sympto-Thermal Method has been demonstrated to be the most reliable method of periodic abstinence $[12,11]$. Although this method still presents some difficulties for charting and chart interpretation, the OC features will facilitate data gathering and will enhance greatly the data interpretation. As part of the STM, BBT changes will be considered an important variable for the detection of the day of ovulation. Many other symptoms will also be added to the OC database which will not take an active role in the automatic decision making, but will be available to the user to confirm results and/or to base any other type of decisions. The combination of STM with the VER and SER methods will likely provide the most reliable and accurate NFP method currently developed.

The first day of the menstrual flow (day 1) will be an important variable, since most of the OC methods will need this parameter as a point of reference for later calculations. 
Finally, the OC will consist of three numerical variables, e.g, BBT, VER, and SER, plus many symptoms that also will be recorded. However, as mentioned before, the OC will be able to accept new variables at any time, with minimum adjustments to the database. 


\section{CHAPTER V}

\section{WHY A COMPUTER-BASED SYSTEM}

The use of computer-based information management systems has increased rapidly in recent years in an effort to adequately process all the patient data being provided by advanced medical equipment. Computer management systems now manage administrative and clinical patient data, images, diagnostic notes and other medical data. Assessments on the usefulness of such data management systems have shown that the data management burden placed on the medical staff is greatly alleviated. The same studies have expressed the favorable acceptance of these computer data management systems by the medical community [31].

In an effort to enhance medical decision support, assessment resources, and diagnosis accuracy concerning the prediction and detection of the ovulation, an extensive research of the latest literature was made, looking for accurate and reliable parameters that can be electrically measured. Some of these methods have been already used for the production of ovulation kits; however, little has been done in an attempt to integrate these methods in a patient database system for use in clinics and hospitals.

There have been very few attempts to build a computer-based system to keep track of the ovulation. In 1984, after field-testing a new set of client forms for monitoring NFP performance in Zambia and Liberia, Action Familiale decided to implement the same forms within its NFP services. However, since Action Familiale recruits about 2,500 new clients annually, it was decided that a microcomputer would be used to handle the data processing needs of the organization. Using various packages made available, Action 
Familiale started computerizing its statistical department as well as other spheres of activities. Unexpected problems at many levels cropped up and prevented full use of the equipment: the electrical supply and equipment and even part of the hardware were not up to local standards; new personnel recruited to work on the computer caused irretrievable loss of data due to a poor back-up system; there were bugs in the software; the questionnaires were not filled correctly; some of the questions were even refused by the NFP department and thus were not asked of the clients. Finally, it was decided that the questionnaire and the whole system would be revised at the end of 1986, and a new approach adopted [32].

In 1981, a group of researchers presented a proposal to Pope John II concerning the design of microprocessor-based device for the detection and prediction of the ovulation [6]. Although good ideas were presented in this document, little acceptance was given to the project. A later study at Portland State University attempted to develop an image processing system to keep track of the day of ovulation based on the Ferning test method. However, results of such a project are still not clear, and lately researchers are not putting emphasis on the Ferning approach anymore.

The main motivation behind the OC stems from the limitations of the traditional paper-based charting system used to record menstrual cycle data. Moreover, the current gathering of ovulation data requires nursing staff to periodically read the data measured through other equipment at the bedside, and maybe even data made available from other departments. This tedious procedure is error prone because of the stressful nature of the patient care [31]. By interfacing the sensors directly to the electronic monitoring system, the OC will automatically and continuously record the patient data, eliminating the burden and errors of the manual procedure. The user will also be able to enter data from the keyboard if necessary. That data could be from other department or any external source. Review and interpretation of real-time data is improved by displaying it as graphical 
trends and charts on a high resolution color monitor.

The development of a computer-based system to help physicians compare and evaluate ovulation detection/prediction methods may appeal to practitioners and researchers in the fertility field. As mentioned before, the diversity of opinions when deciding the accuracy and reliability of rules has been of great concern to physicians and patients [9]. Thus, implementing each of the well known rules in a computer system would allow the physicians to apply a set of data, for example from a cycle, to different rules. In this way, the user would have a series of results from these rules and would be able to evaluate the rules more objectively.

The tendency in recent years has been away from systems that replace the physician or the laboratory technician, emphasizing instead systems designed to assist the medical specialist in making better decisions. Under this perspective, the $\mathrm{OC}$ is considered a tool designed to help nurses and doctors make more accurate diagnosis of the fertile and infertile times. 


\section{CHAPTER VI}

\section{MEDICAL DECISION-SUPPORT SYSTEMS}

Decision-support systems for various applications are a rapidly growing area. Their growth has been made possible by the development of new theoretical approaches and the support provided by advances in programming and in computer hardware. A new area that has been stimulated by this theoretical progress is modern medicine, an area where experts must make decisions in real time under conditions of considerable uncertainty of the observed facts and insufficient formalization of the practical recommendations of medical science [33].

In general terms, Shortliffe defines a computer-based medical decision-support system (MDSS) as a computer program designed to help health professionals make clinical decisions [34]. Regardless of the underlying computer-science methodology, clinical decision-support systems deal with medical data about patients (similar to what would be found in a medical record) or with the knowledge of medicine necessary to interpret such data. They generally help in determining the nature of a patient's disease state or in formulating a plan for reaching a diagnosis or administering therapy. In a sense, any computer-system that deals with clinical data or medical knowledge is intended to provide decision support [34].

Current enhanced opportunities for progress in the field of clinical decision support derive from several sources, including the rapid growth of awareness of and interest in computers and information-management systems, the growing usefulness of medical information systems for helping professionals with other biomedical research and health 
care problems, and the continuing rapid development of the technological base [34].

Only a decade ago, software tools were rarely used by health personnel and were viewed with some skepticism. The change in attitudes has been due in large part to three influences: (1) the emergence of microcomputers and easy-to-use software, with the concomitant demystification of computers and a general sense in society that such machines are manageable and useful; (2) the growing distress among health professionals regarding the amount of information they need to practice medicine well; and (3) the increasing fiscal pressure that encourages the practice of cost-effective medicine, thereby leading practitioners to consider carefully the clinical utility and reliability of tests, procedures and therapies, especially when they are expensive or risky [34].

In order to understand the range of capabilities that computers can provide in assisting with clinical decisions, Shortliffe [34] has classified three types of decision-support functions ranging from generalized to specific:

\section{DECISION-SUPPORT FUNCTIONS}

\section{Tools for Information Management}

These tools provide the data and the knowledge needed by the physician, but they generally do not help non-experts apply that information to a particular decision task. Interpretation is left to the physician as is the decision about what information is needed to resolve the clinical problem. Examples are hospital information systems which provide facilitated access to patient data needed for clinical decision making, and bibliographic retrieval systems which allow rapid access to pertinent information from the current literature. 


\section{Tools for Focusing Attention}

Such programs are designed to remind the physician of diagnoses or problems that might otherwise have been overlooked. They typically use simple logics, displaying fixed lists or paragraphs as a standard response to a definite or potential abnormality. Examples are clinical laboratory systems that flag abnormal values or that provide a list of possible explanations for those abnormalities and pharmacy systems that alert providers to possible drug interaction.

\section{$\underline{\text { Tools for Patient-Specific Consultation }}$}

Such programs provide customized assessments or advice based on sets of patientspecific data. They may follow simple logics, such as algorithms, may be based on statistical theory and cost analysis, or may use numerical approaches. Some examples of this type of systems are Dxplain [35] and QMR which are diagnostic assistants used to narrow the range of possibilities for a particular set of data. Not many systems of this category have been reported so far.

Tools for information management are considered "passive" systems. That is, they make information more readily available to the physician but they do not provide "active" decision support by applying medical knowledge to a specific patient's data, nor do they recommend a specific conclusion or course of action. However, there has been less success in developing active systems. One strategy used in such systems applies predefined rules to the patient's data and alerts providers to conditions that might require action. Systems used for focusing attention present a more active involvement in the diagnosis process.

Active systems contain in-depth knowledge about a well-defined subset of medical knowledge and require considerable and detailed information about the patient's clinical status to provide the computer-generated consultations. According to the three categories 
mentioned before, the OC could be considered an active system. Although the final decision will be made by the physician, the $O C$ automatically analyzes data, and reports all important results based on the command given.

According to Barnett, some important criteria should be considered for a differential diagnosis decision-support system intended for use in routine clinical practice [35]. Such systems should:

- be easy to use by physicians who have little or no computer background;

- be based on comprehensive medical content;

- provide correct and accurate interpretations;

- justify its interpretations;

- evolve and improve as a result of user criticism and analysis of user sessions;

- be convenient to access from the physician's office, hospital, or home.

These features have been carefully considered in the implementation Of the OC system. Remote access facilities is not a important priority of this system; therefore, that point is not considered in this thesis.

\section{MDSS Problems}

Some problems found in MDSS include difficulty in accessing and interacting with the systems, a limited area of application, a limited ability of the systems to explain and justify the interpretations, and less than acceptable quality of the interpretations. Some investigators believe that there are major intellectual and technical problems that must be solved before there can be truly reliable consulting programs. In particular, it is claimed that computer programs must be able to incorporate pathophysiological knowledge and causal relationship based on pathophysiological reasoning. 


\section{KNOWLEDGE-BASED MEDICAL SYSTEMS}

Decision making in medicine is based on two types of knowledge: scientific knowledge and empirical knowledge (experience). Scientific knowledge is acquired in the process of medical research, whereas experience is phenomenological. Empirical knowledge is less formalized, although it plays a dominant role in diagnosis and treatment selection [33].

Recognition of the difference between the two types of knowledge is important for understanding the advantages and the fundamental limitations of the computerization of medical decisions. Let us consider some characteristics of the two types of knowledge.

\section{$\underline{\text { Scientific Medical Knowledge }}$}

This is knowledge acquired by medical research. It does not take into account the complete data about the patient, touching only on certain aspects of the data. As a result, this knowledge is useful only for processing part of the available information. The reason for this is that medical science is concerned, not with the condition of each individual patient, but rather with the general regularities of diseases averaged over a certain group of patients. Scientific medical knowledge is abstract. It is therefore valid within the specific domain of research, and it is inherently limited because of generalization. Models based on scientific medical knowledge also have a generalized and limited character, i.e., they are abstract models.

\section{Empirical Knowledge}

Knowledge of the second type, conversely, is not acquired in scientific research: it is extracted from the actual experience with patients (problems, processes). This empirical knowledge is accumulated in the course of observation of a large number of patients 
under a variety of circumstances. The limitation of empirical knowledge is that the sample of patients observed by the physician may be insufficiently representative of the entire population. Then the accumulated empirical knowledge on average does not fit the population, and the physician will periodically make mistakes [33].

Formalized medical knowledge thus consists of two components:

- scientific theories incorporated in algorithms and computer models;

- medical experience stored in data bases and knowledge bases.

Most of the operating medical decision support systems utilize a combination of the two knowledge components. The purely statistical approach combined with mathematical modeling is an example of using knowledge of the first type, whereas heuristics and expert systems mainly rely on knowledge of the second type.

The methodology of development of medical decision support systems requires resolving the following main issues: choice of the application domain for the system (medicine), collection of well-documented medical data, choice of a source of knowledge acquisition, choice of a decision making strategy or model, choice of parameters for optimizing system efficiency evaluation, and choice of system validation methods.

Of key importance in this list is the choice of decision making strategy or model. A number of approaches have been developed for the modeling of medical decisions. Some of the main approaches are listed below:

\section{$\underline{\text { Statistical Methods }}$}

These methods usually employ the techniques developed for pattern recognition. Statistical methods are mostly used in differential diagnosis. An example of the statistical approach is provided by the use of Bayes' method. If the distribution $P(f \mid D)$ (where $f$ is a symptom) and all the prior probabilities $P(D)$ of the different diseases are known, 
then the optimal decision model is the Bayes rule. This rule actually assumes availability of complete information about symptoms, disease, and their interdependencies. Because of the common differences among doctors, this approach would not be appropriate to implement in the OC.

\section{$\underline{\text { Logic Methods }}$}

Logic decision making uses yes/no decisions, truth tables, or decision trees. Theoretically, statistical and logical decision strategies differ in that the statistical approach uses the collection of features (the feature vector) only once for discrimination, whereas in logic methods the global classification decision is partitioned into a number of sequential partial decisions. The problem is essentially decomposed into a number of connected subproblems. Logic methods assume the availability of a larger volume of empirical knowledge than statistical methods. This knowledge is utilized to construct an adequate decomposition of the original problem. The main difficulty in logic decision making is to establish a reasonable balance between the structure of the decision tree (table) and the content of the partial decisions in the tree nodes (table cells). The main difference between logical and statistical methods is that the former are less formalized and apparently provide less opportunity for model optimization [33].

\section{$\underline{\text { Heuristics, Medical Expert Systems }}$}

Application of decision making often contains numerous heuristic elements. The heuristic approach to decision making has many unexpected diversions and offshoots, which depend on the particular disease and the particular patient, as well as on the choice of the heuristics. The construction of a structured logic tree in these cases is a fairly difficult problem, which has to be solved by artificial intelligence methods. Medical expert systems play a leading role in the development of this direction of medical infor- 
matics [33].

The basic elements of an expert system are the knowledge base, which contains the terminology and knowledge from some field of medicine, mechanisms of inference (decision making) that use the knowledge base, and a module that explains the expert system decisions to the user [33].

The OC and Knowledge-based Systems

Of all the methods mentioned before, the expert system approach better fulfills the requirements in the implementation of a fertility monitor. Although complex and sometimes contradictory, there is a vast terminology and knowledge in the fertility assessment field. In addition, there are numerous established rules that help the decision making process, and that could depend, to some degree, on the same sources of data. The issue concerning the differences among physicians is dealt through the implementation of multiple well-accepted rules, but the final decision is left to the user.

The fertility field undoubtedly contains elements from both scientific and empirical knowledge. However, as far as the establishing of rules is concerned, most of these rules have been based on empirical knowledge accumulated from past observations. The usual procedure is to evaluate large numbers of cycles in order to gather data to evaluate a new method or rule. It is therefore expected that there will be a constant, periodical establishment of new rules. Because this field changes so rapidly, the $\mathrm{OC}$ has been given sufficient flexibility and adaptability to satisfy current and future research and clinical needs. 


\section{CHAPTER VII}

\section{THE OC DATA ACQUISITION SYSTEM}

As a result of the popularity of personal computers more of them are finding their way into laboratories and factories in data acquisition applications. Computers have an enormous influence on the course of measurement, control analysis, and presentation techniques. Computers are also an important tool in the development of data acquisition systems; however, they are not able to produce any productive work without having a user and real-world interface. Making the most of the microcomputers requires an understanding of the computer capabilities, strengths, and limitations. One of these limitations is related to the signals that the computer must process. Most real-world signals (temperature, pressure, flow, speed, intensity, position, etc.) cannot be read directly by digital computers. These parameters are represented by analog signals distinguished by their continuum of levels [36]. Therefore, a translation device is needed to make these signals understandable by the computer.

The OC data acquisition system consists of seven main functions. The Figure 2 shows a block diagram and the interactions among these different parts. 


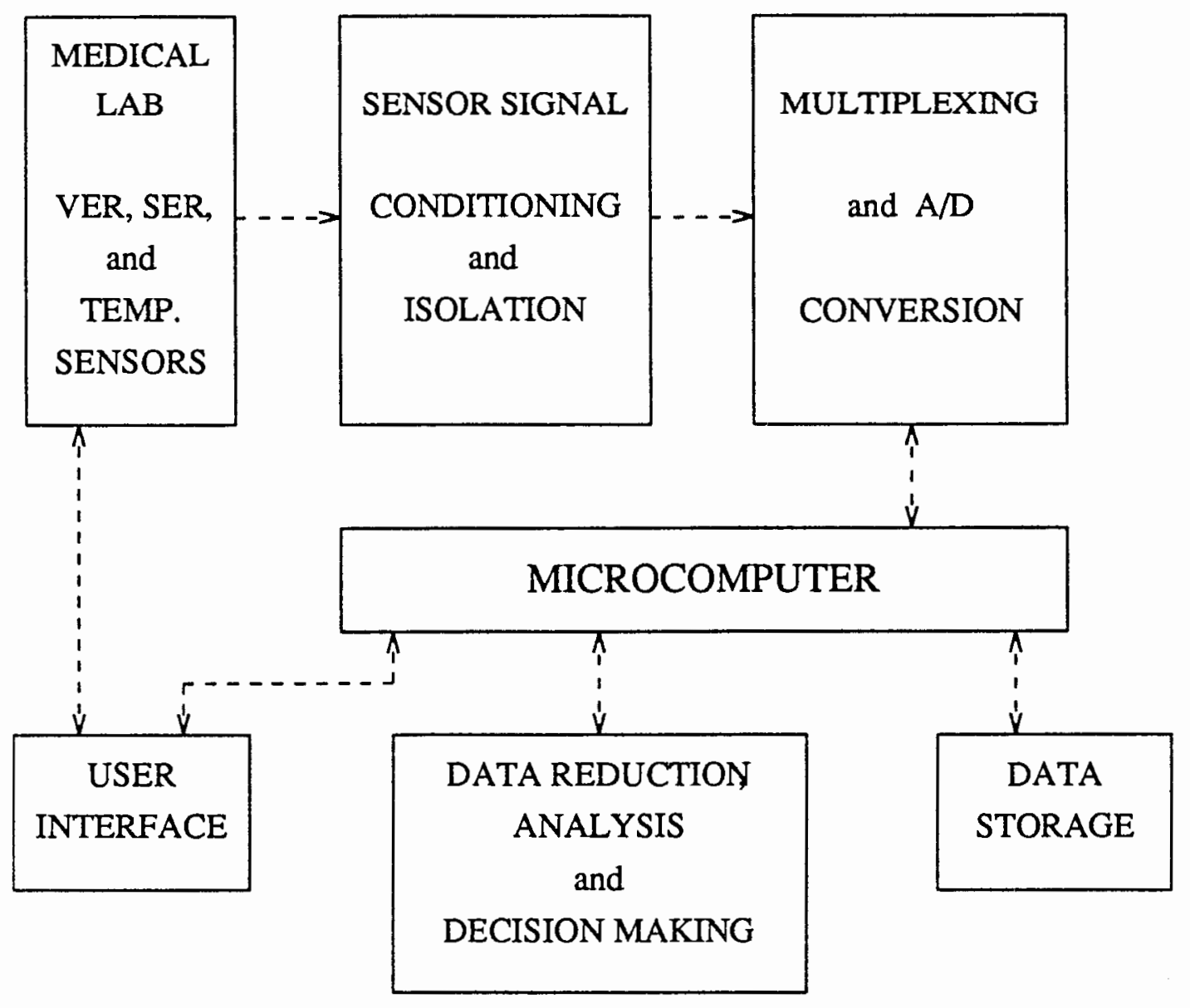

Figure 2. Block diagram of the OC data acquisition system.

The Medical Lab represents the real world the OC will be in contact with in our system. It is there where nurses and physicians will perform different types of measurements directly on the patient. As mentioned before, the OC will offer three automatic options that will collect data directly from the patient, that is, temperature and vaginal and oral resistivity. These sensors will be physically connected to the microcomputer via the A/D converter board. Of course, data can also be entered manually via the keyboard, or through an ASCII file written in the same OC's database format.

The Sensor Signal Conditioning describes the electronics required to convert the varying physical properties of sensors into standard high level signals that can be easily 
converted by standard A/D converters. These functions include: amplification, completion, excitation, filtering, common mode rejection, protection, linearization and cold junction compensation. All these functions are assumed to be part of the electronic sensors that the OC will use. These sensors will not be built. Some medical instrumentation companies have already built VER and SER instruments, such as the CUE monitor, which allow the user to measure both the salivary and vaginal resistivity. Electronic and digital thermometers are much more common and their accuracy, speed, and comfort have been improved considerable in the last years.

Isolation guarantees that there is no electrical (galvanic) connection between the sensor on one side of the isolation barrier and the measurement system on the other side. Isolation can eliminate ground loops and improve safety and reliability of the system. Sensor signal conditioning and isolation are recommended to be performed outside of the computer chassis, with external modules or panels.

The Analog to Digital Conversion is the function that accepts continuous (analog) voltage signals and converts them to digital bits, bytes or words, so that digital computers can read them.

The User Interface is really the human interface to the computer: graphic display, keyboard, mouse, printing capability and software that makes it all easy to use. The user interface allows the user to instruct the $\mathrm{OC}$ what to do, and at the same time informs the user of the results and warnings. Chapters VIII and LX will describe in detail all these techniques.

Data Reduction and Analysis involves using the analytical capabilities of a computer to speed up the process of differentiating between good and bad results. This includes comparing and adjustment of data to desired values, and converting values to engineering units. Data manipulation of all types is done right after the data is received by the program. 
Data Storage is made on hard disk. A backup option could be also implemented to save important data on floppy disks every certain period of time (e.g. every week), depending on the volume and the importance of the data.

\section{IMPORTANT A/D CONVERSION ISSUES}

An important part of any data acquisition system is its host computer. There are two possible ways to interface the data acquisition hardware with the computer, direct connection to the computer bus (internal bus) or via a standard communication channel, such as RS-232, RS-432, or IEEE-488. Each method has its advantages and disadvantages. For the purpose of this project the internal bus connection provided the best choice. Some of these benefits are: computer plug-in boards represent the lowest cost alternative and can gather data at the highest speed. These boards typically have low point counts and rely on the host computer for each operation. They are used in applications where the computer is close to the sensors being measured.

The main advantages of making direct connection to the PC bus include high speed, low cost, and smaller size. Cost is reduced with this kind of $A / D$ converter system because it does not require its own separate enclosure or power supply. Power is obtained from the PC. When the data acquisition hardware resides inside the host computer, important advantages in both size and space utilization are obtained. High speed is achieved by eliminating the relatively slow, external, communications-channel protocol [36].

Speed and resolution are the major considerations of the A/D converter. The actual A/D conversion can be performed by one of three common methods: integration, successive approximation, or parallel (flash) conversion. These methods provide tradeoffs between speed and conversion accuracy. For most applications, 12-bit resolution is sufficient. This provides voltage steps of 1.22 miliivolts for a 5-volt range and 2.44 
millivolts for a 10-volt range. A resolution of 16 bits has smaller steps, but requires more time to execute and is more expensive. Eight bits is coarser and less accurate, but it is faster. Integrating and successive approximation converters can be used for 8-, 12-, or 16-bit resolutions [37].

Other important issues to be considered in the selection of an $\mathrm{A} / \mathrm{D}$ converter are related to the input types and to the data transfer methods. First, input channels can be single-ended or differential. Single-ended inputs have the same reference potential. Differential inputs offer an excellent noise immunity called common mode rejection ratio (CMRR). Differential inputs respond to differences in voltage levels between the two signal leads, and can improve the accuracy where long cables, and low-level input ranges $(<1 \mathrm{~V}$ full-scale) are used. For these reasons, it is strongly recommended the use of a differential-input $A / D$ converter in systems like the $O C$, in which the sensors may be several feet away.

Second, data transfer methods play an important mle when short conversion times are required. In this system conversion speed is not a relevant issue, since a variable such as temperature takes relatively much more time than a $A / D$ converter does. There are three main data transfer methods: programmed 1/O, PIO interrupts, and DMA. The OC would work with any of them. The programmed I/O A/D converters are cheaper, however, slower. Interrupt- and DMA-based A/D converters are faster, relatively more difficult to program, and more expensive.

Finally, another very important part of the OC data acquisition system is the program that controls the hardware of the A/D converter. The data acquisition program cornmunicates with the hardware of the system by using one of the user ports available in the microcomputer. One of the most common causes of failure when trying the board is address contention. Some of the computer's I/O space is already occupied by internal I/O and other peripherals. The A/D converter therefore should have several configurable I/O 
ports in case that some of them are already occupied. This program also is in charge of collecting the data read from the A/D converter. Then, that data is displayed and/or stored in a specific format for later manipulations.

\section{THE A/D CONVERTER USED IN THE OC}

The A/D converter board used in the OC was the AD510 multichannel board built by Real Devices (State College, PENN). This board can be installed in a single or fullsize expansion slot in the microcomputer.

The analog-to-digital (A/D) circuitry receives up to eight single-ended analog inputs and converts these inputs into 12-bit digital data words which can then be read and/or transferred to $\mathrm{PC}$ memory. The $\mathrm{I} / \mathrm{O}$ port used for the $\mathrm{A} / \mathrm{D}$ converter was at the starting address $200 \mathrm{H}$. However, there is up to eight different starting addresses where to choose, which offers great flexibility for those systems that already have some of these ports occupied.

The input voltage range is -5 to +5 volts for a gain of 1 (the range is -0.5 to +0.5 volts for gain $=10 ;-0.05$ to +0.05 volts for gain $=100$ ). The dual slope integrating $A / D$ converter performs conversions at a rate of either 7.5 or $30 \mathrm{~Hz}$, depending on the setting. The $7.5 \mathrm{~Hz}$ rate provides high rejection (over $60 \mathrm{~dB}$ ) of $60 \mathrm{~Hz}$ line noise.

A/D conversions are performed by a dual-slope integrating converter. The output is a 12-bit data word plus a sign bit which tells you whether the digital value represents a positive or a negative voltage. The programmable gains of 1,10 , and 100 let you discern changes in the input voltage as small as 13 microvolts. The converted data is read and/or transferred to PC memory, one byte at a time, through the PC data bus.

The converter samples the input voltage over a window of time before performing the conversion. The converter measures the time required to charge and discharge a 
capacitor. A counter and a reference in the A/D chip determine the integration period. Because the input signal is sampled for a specified period instead of being instantaneously captured by a sample-and-hold device, spikes and glitches which may be present at the input are averaged out. The result is a highly accurate conversion. 


\section{CHAPTER VIII}

\section{THE OVULO-COMPUTER SYSTEM}

One of the first questions asked at the beginning of this project was, how to structure the data, and what programming language or software package to use? As the work went on and the main goals were better defined, we realized that the amount of programming would be very extensive, and to create a flexible system with the capacity to evolve and be improved as a result of user criticism would require an application package such as a spreadsheet.

To make the decision about what kind of software should be used, the following aspects were considered:

- A specific focus of this project was to develop an application for the microcomputer in the field of medicine, rather than software development.

- There is a vast quantity of powerful programming tools available that could serve not only to simplify the work, but also to greatly improve the data management and presentation.

- One of the goals of the project was to exploit as much as possible current microcomputer capabilities both hardware and software, and therefore, the focus was on integrating some of these tools to build up a specific application, a fertility monitor. Some of the hardware tools are, for example, the microcomputer itself, the ADC, the biosensors (CUE monitor electrodes and electronic thermometers). Examples of the software tools applicable to the OC are spreadsheets and high level programming languages. 
There was an initial attempt to develop some programs from scratch in $\mathrm{C}$ language. Although some of the results were satisfactory, issues, such as expandability, user interface and others were restrictive and required a substantial amount of work. Based on some basic knowledge I had on the 1-2-3 spreadsheet, I decided to try to implement the same functions as I did in $\mathrm{C}$ language. As a result, it became obvious that the direction should be the use of a spreadsheet.

\section{THE 1-2-3 SPREADSHEET}

Once the decision to use a spreadsheet was made, some of the following aspects were considered to decide which one. $1-2-3$ is one of the most popular products ever introduced for microcomputers. 1-2-3 has three integrated functions: an electronic worksheet that manipulates numeric information; a database program that stores and can selectively retrieve information; and a graphics program that depicts the numeric data in graph form. The manipulating features of 1-2-3 allow data to be displayed in a variety of ways, and offer users many opportunities to customize.

Combining the power of macros and the autoload feature results in a very userfriendly program that requires, for the most part, only the numeric keypad and ENTER key. Data presentation makes use of the versatile features of the 1-2-3 spreadsheet. The new 1233 -D features allow the programmer to have up to 256 worksheets in one worksheet file. A worksheet consist of 8192 rows and 256 columns. The intersection of a row and a column forms a cell. Each cell can contain one numeric data item, text, or a formula. The width of each cell can be adjusted so that text or data can be clearly seen.

\section{1-2-3 Macro Basics and the OVULO-COMPUTER.}

The OC has been totally based on 1-2-3's command language, which is a full programming language. Its implementation was based on 1-2-3's built-in functions, 
keyboard macros, and command language macros. Briefly described, the built-in functions consist of menus and sub-menus which contain over 100 functions grouped in many categories for a wide variety of specialized calculations. Some of the most useful categories used in the $\mathrm{OC}$ were the ones for database, statistical, time and date, mathematical, and logical operations.

Keyboard macros are essentially columns of label entries that have a name assigned to them. The contents of the labels are sequences of keystrokes that the programmer wants 1-2-3 to execute for him, for example, Page Up, Run, etc. Command language macros are powerful functions that allow the programmer to create custom menus, utilize iterative loops, and alter the order in which commands are executed in response to entries provided by the operator. For more details on 1-2-3 macros refer to the 1-2-3 Reference Manual or any recent book on 1-2-3.

\section{IMPLEMENTED FUNCTIONS}

\section{User Interface}

This was one of the first goals defined at the beginning of the of this project. Although many professionals in medicine have shown a great interest in computers, there is still a great majority who are reluctant to or scared of computer systems.

In response to this need of user-friendly systems, the 1-2-3 spreadsheet was chosen. By using the 1-2-3's versatile custom menu features, the OC user interface has been enhanced considerably. Custom menus allow the user to execute or select any of the OC functions by pressing just the arrow keys and Enter. Each time the user starts the OC the Main Menu appears in the control panel. The control panel consists of the upper three lines on the screen. The user has access to any of $\mathrm{OC}$ functions via the control panel. 
As shown in Figure 3, there are many levels for some of the main functions. For better understanding and guidance, a mode indicator has been added in which are shown the menu depth and title. This mode indicator is located in the upper right corner of the screen. Most of the main functions have help windows to instruct the user of any further procedure, or to warn the user of some careful procedures, especially when accessing patient files. The whole worksheet section, where the programming code is, has been write-protected to avoid any accidental changes. Thus the user is allowed to write or modify data only in the database area, which consists of $\mathrm{n}$ number of columns, one column per variable. 


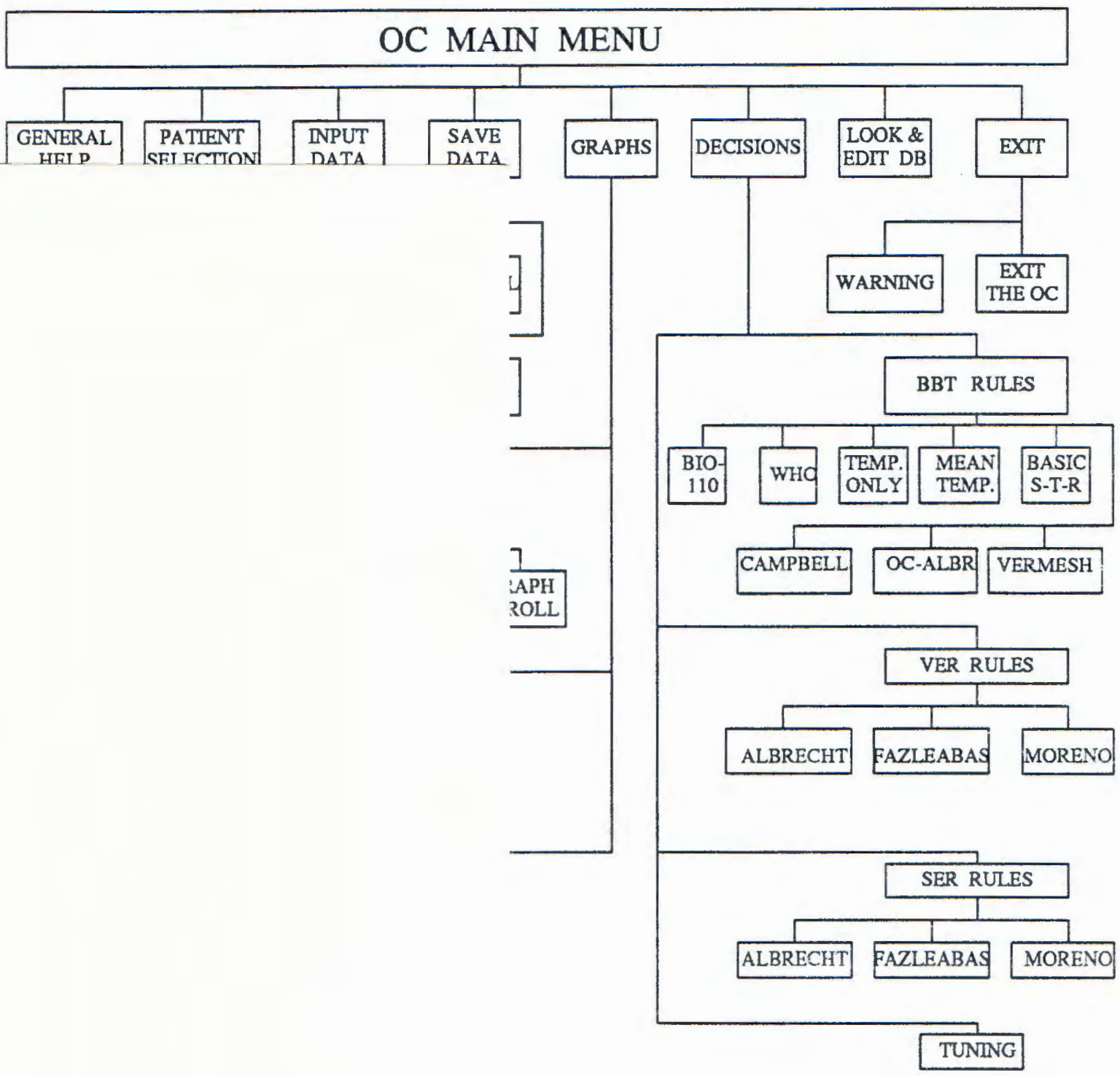

taining all the functions implemented.

Ir interface is greatly improved if a high resolunmands may be facilitated if a mouse is used. step for the improvement of the user interface. Ited so far, may have no meaning for physicians. if a possible problem or error in the data by a 1 a separate screen or in any other styles. There- 
fore, customizing the system may be necessary, process which would not require any major adjustments to the system.

\section{Data Visualization}

In this section the main features that provide the versatility of the OC system will be discussed.

One of the main motivations for building a computerized system to keep track of the status of the fertile period in a woman's cycle was the inherent need for easy-to-use techniques for the visualization of the behavior of the different parameters involved in the decision. To achieve this purpose, the OC, using 1-2-3's features, integrates (in its primary stage) three numeric daily variables: BBT, SER and VER. In addition, the OC has the capability of recording any number of relevant cyclic symptoms helpful in the diagnosis of the fertile and infertile days.

There are two main data presentation styles implemented in the OC. First, data is presented in an organized table style allowing the user to look at individual values in the corresponding units. All of the variables are recorded in consecutive order according to the date when they were entered. The symptoms are also dated and presented in a way which makes it easy to visualize and analyze them.

The other approach in data presentation is in a graphical form. This is probably one of the most powerful features of the OC. Values of BBT, SER and VER are displayed in $x-y$ line graphs. The $x$-axis represents the calendar days. The OC displays the $y$-axis in two different scales. Temperature values are plotted based on the left scale of the graph. The right scale corresponds to the SER and VER values measured in ohms $(\Omega)$. The system automatically adjusts the minimum and maximum values of these scales based on each set of data. The temperature is plotted on a different scale because its range of values (normally from 97.5 to $98.5^{\circ} \mathrm{F}$ ) is too small compared to the range of VER and 
SER, which typically ranges from 70 to 180 units. In this way, the user is able to perceive slight changes in the parameter values and therefore, make more accurate measurements.

The advantage of having the three variables plotted at the same time is essential to the improvement in the reliability of the diagnosis. Physicians and nurses are able to compare and analyze the variable behaviors to either confirm an obvious result, or to detect an abnormality or a special case. As shown in Figure 4, titles, subtitles and comments in the graphs help the user to understand better what is being plotted, as well the interpretation of the graph. If a color monitor is used, each of the curves is plotted in a different color. If the system has a monochrome monitor, curves are distinguished by special symbols that avoid possible confusion or misinterpretation of the data trends. Moreover, the addition of legends greatly enhances the graph presentation. Legends allow the user to add text to the graph that explains what each range in the graph represents. The user might also want to add a grid to the graph to allow a more accurate reading of the data values. Both grids and legends are options accessible in the Graphics-Options menu. 


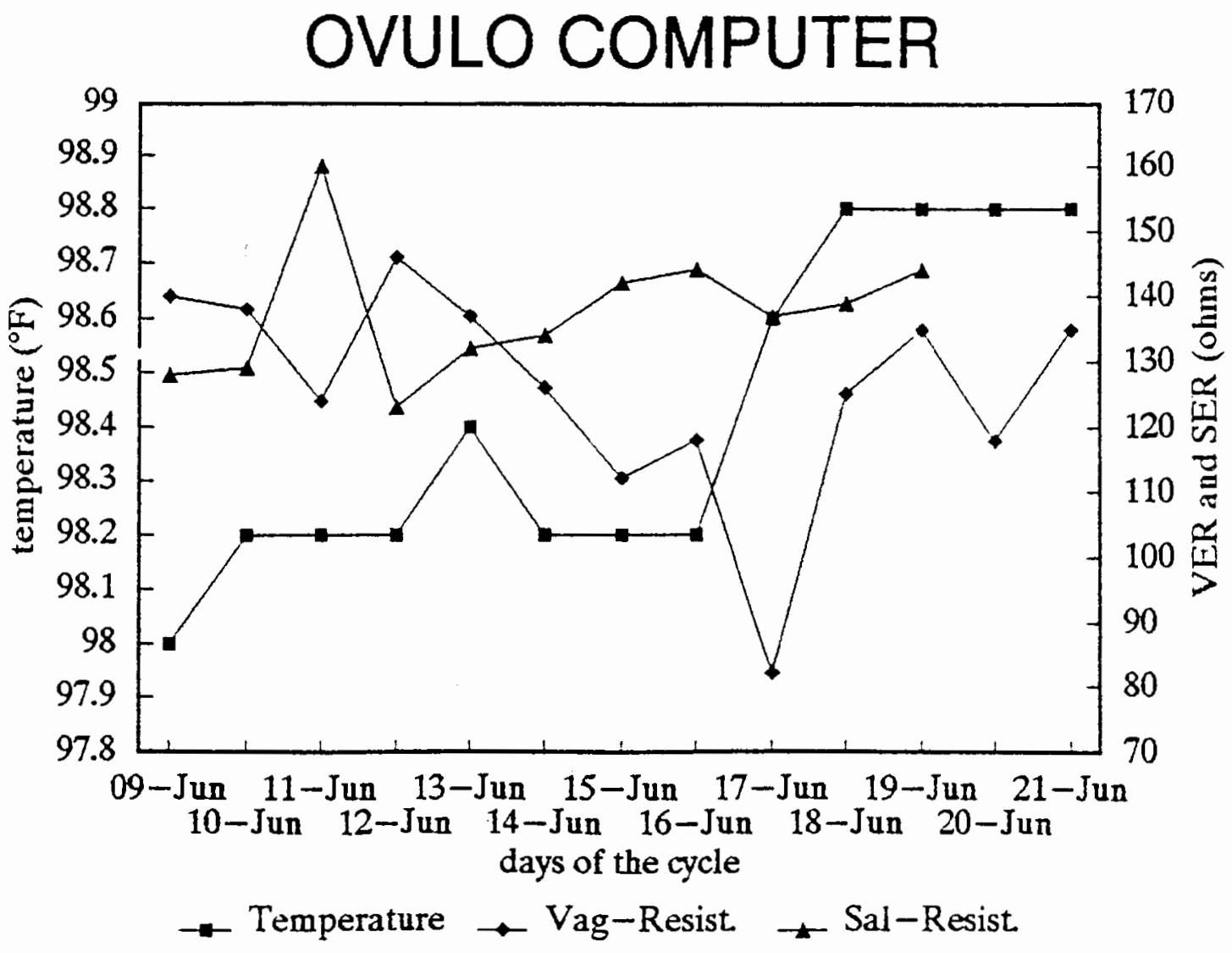

Figure 4. Example of some of the $\mathrm{OC}$ graphic features.

Another special graph feature that increases the flexibility of this system is the ability to display the data ranges both in tables and in graphs at the same time. The screen is split into two windows; the worksheet data is displayed on the left and the graph is displayed on the right. In this special mode, the user can also scroll the data being plotted by just a keystroke. There are also two options to specify how many days to "shift" to the right or left of the displayed window, and how many days to display in that graph window. Shifting a variable in the graph means to offset the independent variable (e.g., days) of the current data range being displayed by a specific value. Moreover, any change or addition made to the worksheet data is immediately reflected in the graph on the screen. 
Database Structure

Data in the $O C$ is organized using the versatile organization of the 1-2-3 spreadsheet. Each single variable was assigned to a column of the worksheet. Columns can grow as big as 8192 entries per worksheet. Rows contain the data of each of variables for each day. Therefore, the total number of days per worksheet is 8192 (about 22 years and 5 months).

Starting with the day, BBT, VER, and SER values and following with the symptoms, the OC can have as many variables (both numeric or text) as desired. Each row of the worksheet corresponds to a day of the menstrual cycle and contains all the data corresponding to that particular day. The leftmost column of the database corresponds to the calendar days. Then, from left to right the following variables and symptoms are displayed: TEMPERATURE, VER, SER, MENSES, CERVICAL MUCUS, CERVIX SIGNS, INTERMENSTRUAL PAIN, INTERMENSTRUAL BLEEDING, BREASTFEEDING, and INTERCOURSE. As mentioned in Chapter III, these variables have been suggested according to recent studies. However, the OC presents great flexibility if the user wants to add new variables or remove any variable that is considered irrelevant.

The main reference variable of the system is the calendar days. However, the database has also an added variable called "MENSES" which is defined as the first day of the menstrual cycle. That data is supplied by the patient and must be entered in the corresponding calendar day. The first day of the cycle is a very important variable, since many of the decisions and algorithms in the OC depend strictly on the cycle days and not on the calendar days. When data is entered using the automatic data acquisition (described later), the OC will leave space for missing data and allow the user to add the data at a later date. 
Automatic Data Acquisition

Automatic data acquisition consists of two main parts. As mentioned in Chapter VII, data is first acquired directly from the patient by the analog-to-digital converter. Then data is transferred to a file. This procedure will be described later. Once the data is stored in an ASCII file, the data is acquired by the 1-2-3 worksheet by executing the command Input-Data-Automatically. This command automatically inputs and adjusts the data into the worksheet format. However, the user will be asked if $s /$ he wants to enter each individual value into the database. This is particularly useful when more than one measurement is made in the same day. The has the option to keep the previous values or to update them.

Error Checking. Once the data is read from the file, it does not go directly to the database. First, the data is checked to see if there is any evident error in the data values, e.g., values too high or too small compared to typical values. If an error is detected, the $\mathrm{OC}$ beeps warning the user of a potential problem in the data acquisition procedure of that variable. If one or more of the values read from the sensors is right, the user has the option to add only that particular variable and omit the incorrect ones.

\section{$\underline{\text { Manual Data Acquisition }}$}

This option gives the user the facility to input data from the keyboard. This data may be from historical records kept on ovulation charts or from any other medical department. This data acquisition mode is especially useful when the patient, for any reason, is not available for the measurements corresponding to that day. Parameters such as temperature or any symptom, which are easily kept at home, could be later entered into the database.

Also, data could be entered into the main database if it were recorded in an ASCII file under the same format used in the $\mathrm{OC}$ database. This format consists of numerical or 
text values separated by commas. Each line corresponds to one day. The first variable of each line is the temperature value, since the OC provides the date. The user needs to position the cursor on the day corresponding to the first measurement recorded in the ASCII file. This option could be especially useful for hospitals and clinics that want to transfer historical records to the $\mathrm{OC}$ database. Once the person typing this data knows the format, the only requirements would be a standard PC microcomputer and an ASCII text editor. 


\section{CHAPTER IX}

\section{DECISIONS AND RULE IMPLEMENTATION}

The OC, in addition to providing excellent visualization of parameters, also has the ability to make decisions that could help nurses and physicians to make a more accurate diagnosis of the fertile and infertile days. The OC could be considered an "intelligent" system in the sense that it presents knowledge taken from rules widely accepted by prominent gynecologists.

Because of the nature of the medical field, it is very difficult to find total or even partial agreement among doctors as to the methods, procedures or considerations of their studies. To alleviate this potential problem, the OC utilizes knowledge from the most reliable and accurate methods currently being used or studied. The user is able to apply any of those well-known rules to any particular woman's cycle. Also, there is an option which considers all the rules together if sufficient data is available. A description of each of these rules implemented in the OC is given in the next three sections.

\section{RULE DEFINITIONS}

\section{$\underline{\text { BBT Rules }}$}

All the BBT rules implemented in the OC depend on measurements of the temperature at least from the Day 6 after the onset menstrual flow. For Campbell's rule, temperature values have to be taken from the fourth day of the menstrual cycle. Missing values are interpreted differently by each rule. Taking good temperature measurements from 
day 6 of the cycle until the ovulation day is detected, is crucial when accurate decisions are required. Some of the figures added illustrate the most critical values that have to be considered to make accurate decisions.

WHO's Rule [9]. The World Health Organization's definition of changes in BBT indicates that a shift in BBT to the hyperthermic phase of the cycle should occur within a period of 48 hours or less. Three consecutive daily BBTs should be a least $0.2^{\circ} \mathrm{C}$ higher than the average of the previous six daily temperatures [9]. Missing values are interpreted as "don't care". However, when more than one value is missing the rule does not guarantee an accurate decision. Figure 5 shows the six previous values before ovulation happen, and the consecutive high values ("highs") which determine that ovulation just happened.

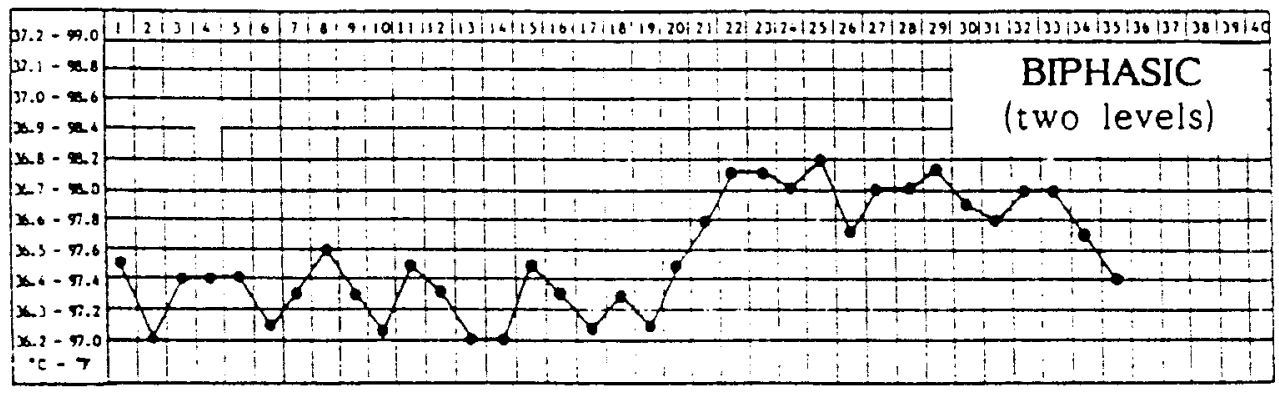

Figure 5. Critical values as considered by WHO's BBT rule.

VERMESH's Rule [2]. BBT has to be measured from the day 5 of the cycle. The temperature charts are interpreted with the nadir (lowest point on the temperature curve) as the predictor point of ovulation. The BBT nadir is the lowest point on the curve, followed by two or more consecutive rises, of which at least one is above the baseline. Ovulation will occur in any of the next three days [2]. A missing value around the day of ovulation may result in no detection of the ovulation for that particular cycle. An example of the type of cycle detected by this rule is shown in Figure 6. 


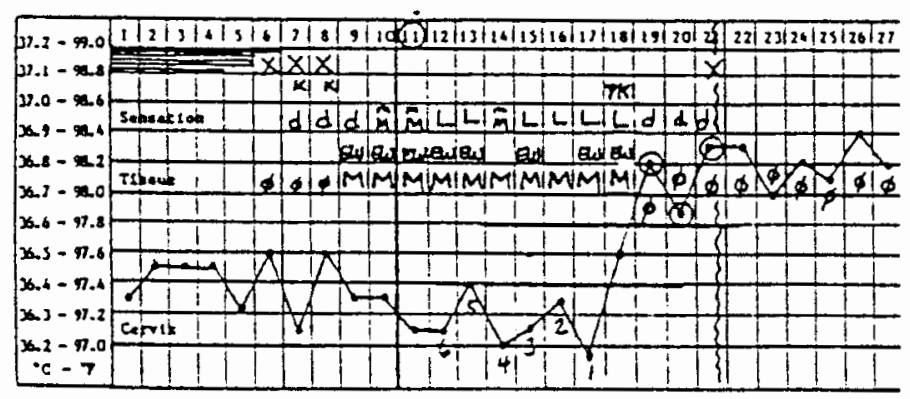

Figure 6. Critical values as considered by Vermesh's BBT rule [12].

BIOSELF 110's Rule [24]. This algorithm requires eight temperature measurements before it can identify the temperature shift from the preovulatory level to the higher level which follows ovulation. In a run of eight consecutive temperatures, the last three temperatures are compared with the first three temperatures, skipping the middle two readings. The three higher temperatures, individually and combined, must be higher than the three temperatures against which they are being compared by a specified amount. Missing data may not be as critical as in other rules. However, it may also result in no detection of the start of the infertile time.

CAMPBELL's Rule [4]. Algorithms for prospectively interpreting BBT begin by establishing a baseline, e.g., the average of the temperature from days 4-11 after the beginning of menses. A coverline, or reference line, is drawn $0.05^{\circ} \mathrm{C}\left(0.1^{\circ} \mathrm{F}\right)$ above this baseline and serves to approximate the upper bound of a confidence interval allowing statistical comparisons between the baseline mean and the subsequent, daily temperature measurements. A rise above the coverline signals the recent occurrence of ovulation and allows computation of the end of the fertile period [4]. Missing data is not taken into consideration. However, if many days are missing the average value obtained may be considerably changed. 
Basic Sympto-Thermal Rule [12]. To fulfill this rule the woman has to chart both the temperature and mucus observations. There are several steps: See Figure 7. (1) Set the pre-rise base. This is the highest of the final six lows before the thermal shift. (2) Determine the peak day. Peak is the last day of any trait of egg-white mucus. (3) Draw a "full thermal shift level" line $0.4^{\circ} \mathrm{F}$ above the pre-rise base. (4) If the third reading after the mucus peak is higher than the full thermal shift level line completely infertile time starts that evening. If the third temperature value after the mucus peak is below the full thermal shift level line, wait until evening on the fourth high reading before assuming completely infertile time. Missing data may vary the pre-rise baseline, but the rule still provide a decision.

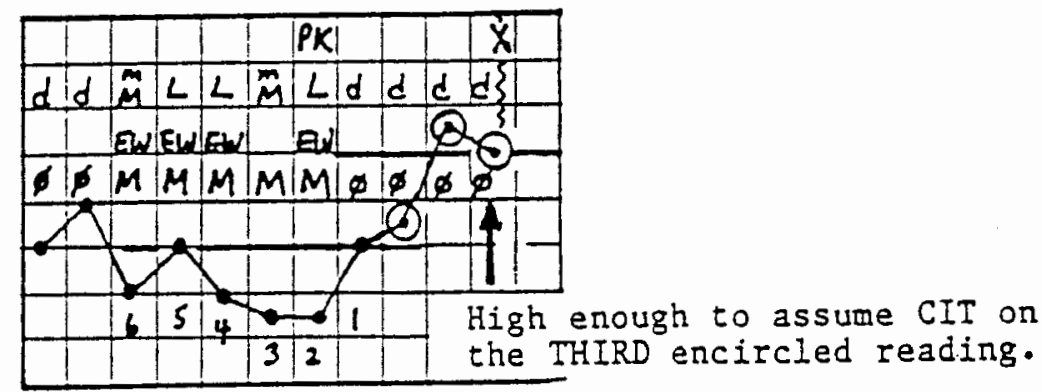

Figure 7. Example of the critical values considered by the Basic STR [12].

Temperature-Only Rule [12]. This rule looks for three high values in a row all 0.4 ${ }^{\circ} \mathrm{F}$ or more above the pre-rise base. The three values have to be consecutive as shown in Figure 8. Completely infertile time begins on the evening of the third high value. As in the Basic STR, missing data may affect the value of the pre-rise base, but the rule still provides a decision. 


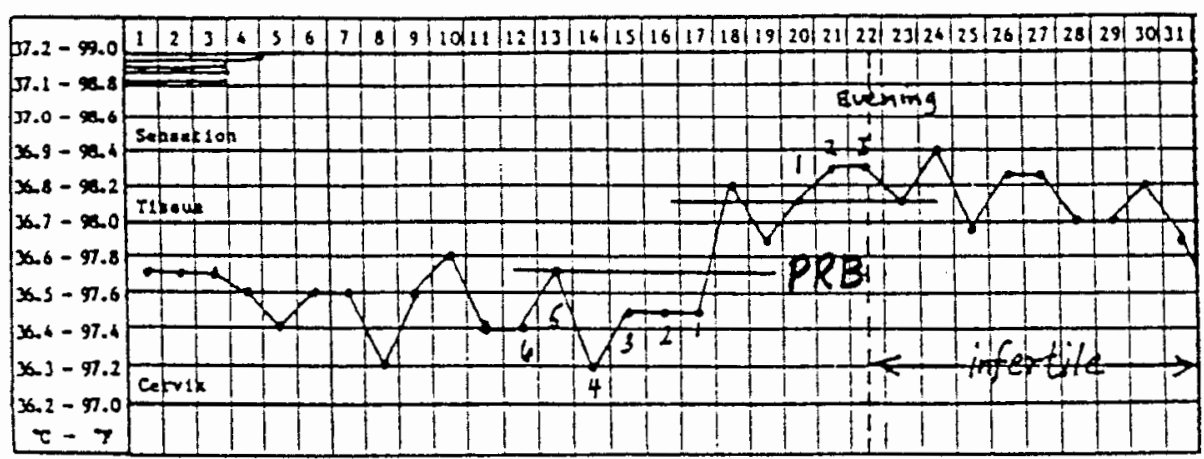

Figure 8. Example of the critical values considered by the Temperature-Only rule [12].

Mean temperature Rule [12]. This rule uses the mean (average) temperature of one cycle as the dividing line between lows and highs for the following cycle. Therefore, daily charting has to be done in order to use that information for the next cycle. Completely infertile time begins in the morning on the fourth high temperature value in a row. Missing data may result in delaying the start of the infertile time.

OC-Albrecht's Rule [16]. Based on observations in some of the BBT cycles and according to some general statements reported by Albrecht [16], a new rule for the estimation of the possible day of ovulation was established. This rule detects the day of ovulation by identifying a shift of $0.2{ }^{\circ} \mathrm{F}$ or higher than the average of the previous six daily temperatures. Figure 9 illustrates the average line. The Dashed vertical line indicate the estimated day of ovulation. Missing data around the day of ovulation may result in detecting the possible day of ovulation one day after the day it actually happened. 


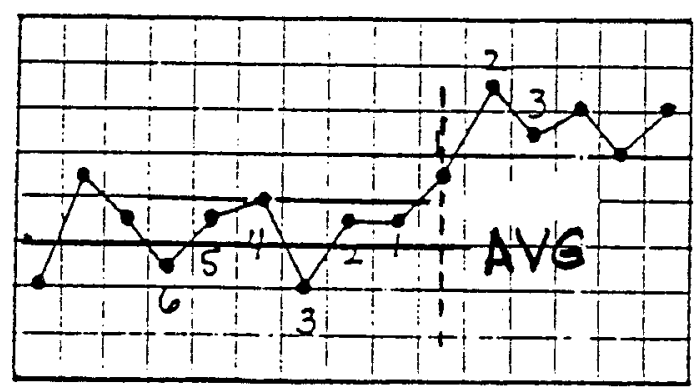

Figure 9. Average line and estimated day of ovulation as determined by OC-Albrecht's rule [12].

\section{VER Rules}

All VER rules are based on very similar criteria and are illustrated by Figure 10. Making good measurement around the day of ovulation is essential for all these rules. A missing data around the VER nadir day may result in no detection of ovulation for the particular cycle.

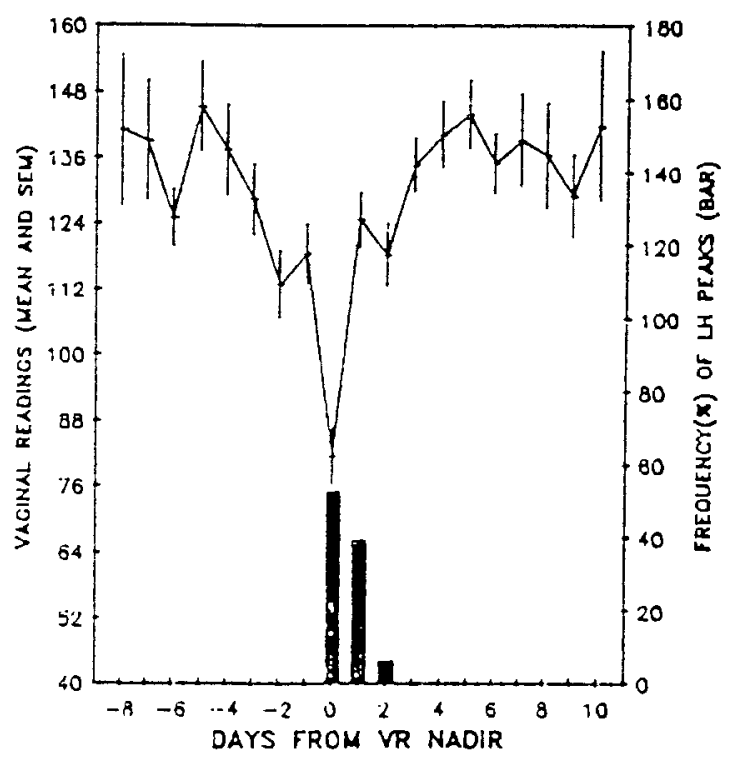

Figure 10. Typical VER values around the day of ovulation. The black bars show the timing of the LH peak [18].

ALBRECHT's Rule [16] According to Albrecht's results a clearly defined nadir of the VER values was found on the LH peak day. The nadir value constitutes an independent value, that is, there is a minimum value ( 68.5 units) that could be used as a reference 
value, since the VER values before and after this day are significantly higher. Besides this reference value, there is a marked change during a 24-hour interval occurred between the VER nadir day and the next day. This rise is about $70 \%$ (48 units) higher than the VER nadir value.

FAZLEABAS' Rule [17]. The nadir of VER was defined as the low vaginal reading, observed four or more days after the SER peak, and just prior to a sustained significant increase [17]. See also Figure 11.

MORENO's Rule [15]. A vaginal nadir is defined as: a decrease in vaginal readings with a subsequent increase for at least 4 consecutive days. Therefore, the vaginal nadir cannot be determined until at least four days after it has occurred. The fourth day after the nadir has occurred is thus called the VER "signal". In certain cases, the vaginal readings in one of the four consecutive days may not show a continuous rise. A plateau or slight decrease may be demonstrated. The vaginal nadir will still be defined by the previous lowest reading as long as subsequent readings do not approach within 20 units of this nadir [15].

\section{$\underline{\text { SER Rules }}$}

Figure 11 illustrated a typical behavior of the SER values in woman's menstrual cycles. Making good measurement around the day of ovulation is essential for all these rules. A missing data around the SER peak day may result in no prediction of ovulation for the particular cycle. 


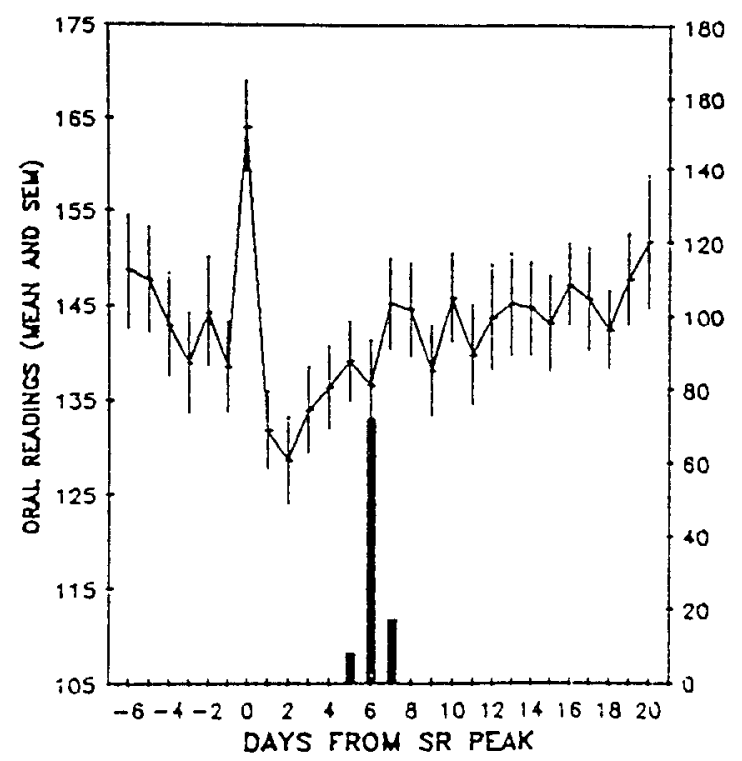

Figure 11. SER values around the day of ovulation. The black bars show the day of ovulation as estimated by LH analysis [18].

ALBRECHT's Rule [16]. The first peak in SER values from each cycle, occurring on or after the fifth day of the menstrual cycle, is defined as stage A. SER peak is defined by a gradual increase in SER for several days and then a sharp declined after the peak value. Then the SER values stay low for three days after stage $A$, at which time it is approximately 40 units lower than stage A. Beginning on the third day after stage A, values increase for several days [16].

FAZLEABAS' Rule [17]. The SER peak is defined based on the rule used by Moreno with a slightly difference. The SER peak is a high oral reading occurring on or after cycle day 5 and followed by lower readings on the two succeedings days, with a cumulative difference from the proposed peak of $12.5 \%$ or more [17].

MORENO's Rule [15]. The following rules are used to define the oral salivary peak: 1) A rise in SER readings with a subsequent drop for at least 2 consecutive days for a minimum of 20 units. Therefore, the oral peak cannot be determined until at least two days after it has occurred. The second day after the peak has occurred is thus called the "Signal". 2) A one-day decrease with a subsequent rebound could also define the peak as 
long as the decrease is substantial and the rebound does not approach within 10 units of the peak [15].

Because of the accuracy and type of information provided by the symptoms, none of the rules were based on them. Symptoms are important secondary indicators that can help physicians confirm results obtained by other methods. They need a special type of interpretation which is difficult to code and whose information when added to the diagnosis is not very relevant. The symptoms of beginning of menstrual was used to determine the first day of the cycle.

All of these rules have been implemented using the set of instructions of the 1-2-3 Command Language. Instructions such as, if ... then ..., custom menus, and function calls were some of the most useful for the implementation of the rules. Each sets of rules (e.g. BBT, VER, and SER) has been implemented and organized in menus and submenus. 


\section{CHAPTER $\mathrm{X}$}

\section{PARTIAL EVALUATION OF THE OVULO-COMPUTER}

Testing of the system with real data is undoubtedly one of the most important parts of any evaluation. Since there is not enough time to evaluate this system in a hospital or clinic setting with real-time data, all the data used was taken from fertility journals. In fact, most of the papers from which the rules were taken also proved to be important sources of data.

The BBT method was the most thoroughly tested, due to the existence of more data reported for this variable. A clear advantage of the data used was that, in addition to BBT values, many other parameters (e.g. LH or urinary assays) were also measured concurrently. That provided a point of reference against which to compare our results. As shown by the average of the days after ovulation in Table 1 , some sets of data differed from each other. Although each cycle demonstrated a cyclical behavior, many ranges of values were found among them. That became a positive aspect of the evaluation, since the system was tested by exposure to many different types of real data.

Table 1 shows a summary of the results obtained after testing each rule for all the available cycles. Nineteen cycles were used in the BBT tests. The left column corresponds to the source of the data. The source data are represented by the author last name, and in some cases, the page number when more than one cycle was taken from that author. Also, the reference number is provided. The leftmost columns correspond to the results of the rules used to determine the start of the completely infertile time (CIT). The right most columns represent the results of the rules used to detect the possible day 
of ovulation (PDO).

\section{TABLE I}

\section{TESTING RESULTS FROM EIGHT BBT RULES APPLIED TO THE SAME SETS OF DATA}

\begin{tabular}{|c|c|c|c|c|c|c|c|c|}
\hline \multicolumn{9}{|c|}{ OVULO COMPUTER TESTING RESULTS } \\
\hline & \multicolumn{5}{|c|}{ COMPLETELY INFERTILE TIME RULES } & \multicolumn{3}{|c|}{ RULES FOR DETECTION OF OVULATION } \\
\hline DATA SOURCE & B10-45 & Blo-s & wно & TMP-ONLY & BSTR & VERM & CAMP & OC-ALB \\
\hline Laurie[26] & 2 & 3 & 2 & 3 & & & 0 & 0 \\
\hline Laurie[26] & 2 & 2 & 2 & 3 & & & 0 & 0 \\
\hline Laurie[26] & 3 & & 4 & 4 & & 0 & 0 & 0 \\
\hline Lanise[26] & 4 & 4 & 6 & 6 & & 2 & 0 & 0 \\
\hline Lanrie[26] & 2 & 6 & 2 & 3 & & 1 & 0 & 1 \\
\hline Albrecher $[16]$ & 2 & 3 & 3 & 4 & & 5 & 0 & 0 \\
\hline Huneger[12] & 2 & 2 & 2 & 2 & 2 & 0 & 0 & 0 \\
\hline Huotgar [12] & 2 & 3 & 3 & 3 & 2 & 0 & 0 & 0 \\
\hline Huneger[12] & 1 & 2 & 2 & 2 & 2 & -3 & 0 & 0 \\
\hline Huneger [12] & 2 & 2 & 2 & 4 & & 3 & 0 & 0 \\
\hline Huneger[12] & 2 & 2 & 2 & 3 & 2 & 0 & 0 & 0 \\
\hline Hureger [12] & 1 & 2 & 2 & 4 & 2 & & 0 & 0 \\
\hline Huneger [12] & 1 & 1 & 2 & 3 & & 0 & 0 & 1 \\
\hline McCarthy[9] & 1 & 2 & 2 & 6 & & 0 & 0 & 0 \\
\hline Hudeger [12] & 2 & 2 & 2 & 2 & 2 & 5 & 0 & 0 \\
\hline Hunegor $[12]$ & 2 & 2 & 3 & 3 & & & 0 & 0 \\
\hline Hurueger $[12]$ & 2 & 3 & 3 & & 4 & 0 & 0 & 0 \\
\hline Huneger[12] & $I$ & 1 & 1 & 2 & 2 & 0 & 0 & 0 \\
\hline Huneger (12) & 1 & 1 & 2 & 2 & 2 & & 0 & 0 \\
\hline Avg of of daynafter ov. & 1.842 & 2389 & 2473 & 3.277 & 2222 & 0.928 & 0 & 0.105 \\
\hline Cycles deructed & 19 & 18 & 19 & 18 & 9 & 14 & 19 & 19 \\
\hline Stendend Doviacion & 0.744 & 1.161 & 1.045 & 1.193 & 0.628 & 2.086 & 0 & 0.307 \\
\hline
\end{tabular}

Evaluation of the rules used to detect the PDO confirmed the reliability of two of the rules implemented. First, a total agreement was found between results obtained with Campbell's rule and the results reported by many authors. This rule was able to detect the PDO in $100 \%$ of the cycles tested. Because of its consistency and accuracy compared to many studies, Campbell's rule became a reference used to test other implemented rules. Second, important results were also obtained by using OC-Albrecht's rule. As defined in Chapter IX, this rule proved to be an accurate detector of the PDO. This rule correctly detected the PDO in 17 out of 19 cycles tested (90\%) and the PDO for the 
other two cycles was detected a day after Campbell's rule. This rule also detected the PDO in all the cycles tested. These two rules provided some of the most relevant results in our evaluation of BBT as an ovulation detector.

Of all the rules implemented, the most unreliable and inaccurate was Vermesh's rule. This rule was implemented with the purpose of identifying the PDO. However, in only 14 of the 19 cycles was the day of ovulation detected and only in 8 cycles did it indicate the day correctly. These results are in agreement with many studies that have shown that a BBT nadir on the day of ovulation is an unusual event [38]. This rule was considered one of OC's rules for those cases in which a woman's cycle may present that pattern; however, its inaccuracy may considerably restrict its usefulness.

There were also significant results obtained in the detection of the start of the CIT. As a general rule according to some authors, CIT starts in the evening of the day detected. One of the most reliable of these rules tested was the BIOSELF rule. There were two different approaches taken using this rule. The BIO-0.45 version appears to give a better performance than the BIO- 0.5 rule. However, as shown in Table 1 , the average number of days after ovulation is not high enough to assume that CIT has started. With a factor of 0.5 or higher between the three lows and three highs being compared, the average number of days after ovulation increases enough to assume CIT has occurred, according to the literature. The reason these two BIOSELF versions were added to the OC was to provide a variety of choices for the experts. Researchers may be able to make this rule more accurate and useful by changing this comparison factor. Actually, the user is able to choose any comparison value for this rule, by accessing the Decision/Tuning option. However, it is recommended that this value be kept in the range between 0.3 and $0.6^{\circ} \mathrm{F}$.

WHO's rule showed an excellent overall performance. An average of $2.5(\mathrm{SD}=1)$ days after ovulation makes this rule one of the most reliable and precise BBT rules for 
determining the start of CIT. Only in one of the 19 cycles was CIT detected one day after the day of ovulation.

The Temp-Only rule was the most "conservative" of all the rules. The average number of days after ovulation was $3.28(S D=1.2)$. This is probably one of the safest of all the CIT rules; however, it may probably delay the start of the CIT by one more day of abstinence than the other rules.

The Mean-Temp rule, by definition, has an additional requirement; it uses the mean-temperature of the previous cycle. This rule has a drawback. Women must take their temperature every day in order to obtain a precise average of previous cycles against which comparisons are made. Since complete and consecutive cycles are usually not reported in the literature, testing this rule was not possible.

Probably the most reliable and accurate rule of all the CIT rules is the Basic-STR. As shown in Table 1, except in one cycle, the CIT was determined exactly two days after the day of ovulation. There is a cost however, which involves making observations not only of the temperature, but also of the cervical mucus, either sensation or tissue-paper observations. For the purposes of rule implementation, the Basic-STR is very similar to WHO's rule; however, the user has to crosscheck by looking at the symptoms which indicate that the cervical mucus peak occurred at least three days before the CIT day is determined.

Testing data for VER and SER methods is scarce. As mentioned before, these are very recent methods and not many evaluation studies have been done. Fortunately, most of the studies published so far confirm the validity and reliability of these methods. Because of the simplicity of the rules for the evaluation of SER and VER variables, results from testing VER and SER techniques were in total agreement with the literature. Five sets of data for VER and four for SER were used. Each of these sets of data represented a great number of women's cycles. In all cases, the SER peak and the VER 
peak were detected. Obviously, a more exhaustive evaluation must be made for these methods. Some differences in the data ranges for each cycle are due to the calibration of the CUE monitor for each study. However, there was consistency in the relative changes in each set of data, which is essential for the OC rule implementation. 


\section{CHAPTER XI}

\section{METHODOLOGY FOR THE EVALUATION OF THE OC}

\section{PROPOSAL}

The primary purpose of this chapter is to develop an objective methodology for the evaluation of the validity of the $\mathrm{OC}$ as an accurate fertility monitor, and as a system that improves the reliability and effectiveness of some methods for the detection and prediction of the ovulation, by automating many error-prone procedures.

Two hypotheses are proposed for this study:

1- The effectiveness of the rules implemented in the OC will be greater than that using traditional rule interpretation methods.

2- The OC will simplify the decision-making process by assisting nurses and physicians with automatic procedures.

The general structure of the study will consist of an experimental group and a control group. The control group will act as a reference to compare the OC's performance in the experimental group.

Ideally, independent experiments should be made to prove or disprove each of the hypotheses. However, since these two experiments would be so closely related, this study attempts to use the same data in a single extended experiment. In order to evaluate the first hypothesis, two parameters will be measured in both groups:

- Accuracy of the decision made, based on a reference method,

- Number of unplanned pregnancies during the study. 
This study proposes the determination of accuracy using the data of three of OC's variable, BBT, VER and SER as follows: For prediction of the day of ovulation for birth control purposes only, the SER variable will be used. To detect the day of ovulation, VER and SER will be used. To detect the start of the infertile time, VER and BBT will be used.

A coordinating committee should be selected for the process of evaluation in each group. Such committees attempt to add a high degree of impartiality to the study. In the experimental group, the committee must work closely with someone who knows the OC system well. This committee should be formed of nurses and physicians, and an engineer who can assist in technical issues related to the specific details of the OC. The committee for the control group could be formed of nurses and physicians, although NFP instructors and practitioners could also be part of it. Each committee is in charge of teaching the correct procedures and instructions to the rest of the staff involved.

\section{Selection of Participants for Both Groups}

Experience indicates that each of the two groups should contain at least 25 patients and be equal in size [39]. The allocation of patients will be randomized to avoid possible bias. At the beginning, none of the volunteer women will know which group they will be in. Each participant must be notified of possible risks such as unplanned pregnancies. Participants should also be informed of the confidentiality of the data and the length of the study. In this study, the age range of the patients will be from 19 to 40 years of age. The length of the study as reported by many should be a minimum of 14 cycles $[2,17]$.

\section{Procedures and Measurements}

In order to compare each of the decisions for each method in both groups, a reliable way to detect the day of ovulation is necessary. The urinary LH concentration as meas- 
ured by the Ovustick kit has proven to be a very reliable way to detect the ovulation day $[2,40]$. It also has the advantage of not requiring clinic visits, since tests can be made easily at home.

Women should not be influenced by any type of information provided by the Ovustick kit. To avoid that women will not be instructed on how to interpret the data provided by this kit. A LH surge guide is necessary in order to interpret the data. This guide will not be give to the women in the experimental group. Procedures on how to use this kit and on the interpretation of the data have been reported in literature [2]. Both the experimental and the control group will use the Ovustick as a reference for the day of ovulation.

This study will be based on the rules as defined in Chapter IX. Each woman in the control group will be given a CUE Monitor for VER and SER measurements and a thermometer for BBT measurements. Procedures for VER and SER have been described clearly in the literature [16]. Participants in the control group will be taught how to use the sensors and devices, and how to interpret the data. Charts will be given to participants to record each daily measurement, as well as the decisions made in each cycle. To keep data more organized and to avoid possible confusion in data interpretation, each BBT, VER, and SER values should be charted on separate charts. Charts should at least provide an $\mathrm{X}$-axis scale for each variable and the cycle days on the $\mathrm{X}$-axis.

The time of the day for each of these measurements must also be standardized. According to Albrecht's study, measurements of SER and BBT should be made soon after the patient awakens in the morning, before eating or smoking [16]. Albrecht also recommends that VER measurements be taken during the late afternoon. Most of these rules require data collection starting on day 4 of the cycle.

In order to extract data for the evaluation of the $\mathrm{OC}$ as a helper in the decisionmaking process (second hypothesis), each member of the control group will be asked to 
use the $\mathrm{OC}$ for data analysis and decision-making procedures. This procedure will be performed at the end of the study when the experimental group has finish the data collection process. Thus, the BBT, VER, and SER measurements already stored in the OC by the experimental group will be used again for both groups. The control group committee members, therefore, will provide important information for two purposes:

- For the interpretation of the control group patient's data (part of the first hypothesis)

- To evaluate the usefulness of the OC (second hypothesis).

To obtain good statistical significance, the control group committee should have as many members as patients in that group. To evaluate this hypothesis some questions have been proposed:

- Is the decision-making process faster using the OC?

- Is it easier to make conclusions about the status of fertility using the OC?

- Does the OC help the user interpret data obtained from difficult cycles?

- Is the OC too difficult to operate?

- Should the OC's user interface, speed or usefulness be improved?

\section{Control Group Committee Tasks}

- Teach the control group patients the procedures for using the different types of sensors and devices.

- Teach patients how to collect data and interpret the data based on BBT, VER and SER methods.

- Evaluate the decisions made by the patients, using the reference method (Urinary LH peak).

- Provide information for the evaluation of the second hypothesis.

- Provide assistance and follow-up to the patients. 


\section{Tasks of the Experimental Group Committee}

- Standardize all procedures concerning data collection and interpretation.

- Train each each of the committee staff members of the experimental group.

- Schedule all patient visits to the hospital or clinic where measurements are held.

- Collect and interpret data using the OC.

- Compare and evaluate decisions made with the OC against the reference method.

$\underline{\text { Results and Data Analysis }}$

At the end of the study each committee should have the BBT, VER, and SER data recorded in charts for the control group, and stored in the $\mathrm{OC}$ for the experimental group. Now each committee must analyze and sort the data. Before making any statistical analysis of the data, the committees should review each cycle for each participant and determine if each cycle qualifies for the study. Some cycles may be incomplete or may represent special cases that should be considered differently. Analysis of the dropout rate and the possible reasons is recommended.

Once all data have been sorted and reviewed, each committee is ready to perform a statistical analysis on the data collected. To evaluate the first hypothesis, the experimental group will be compared with the control group using the following parameters.

- The accuracy of ovulation detection using VER

- The accuracy of ovulation detection using SER

- The accuracy of the detection of the start of the infertile time using VER and BBT

- The number of unplanned pregnancies.

To determine the accuracy of the information provided by BBT, VER, and SER, decisions made before the actual day of ovulation, as detected by the Ovustick, will be represented in negative numbers. Decisions made on the day of ovulation will be represented by 0 , and decisions after the day of ovulation will be represented by positive 
numbers. For example, a decision made 5 days before the actual day of ovulation will be represented as -5 for statistical analysis purposes. This study recommends using the mean and standard deviation of the decision days detected in all the cycles.

To evaluate the second hypothesis, the percentages of the number of people who answer the question will be used. Since each answer has has been scored using a scale of 1 to 5 , it is recommended that the number of answers to each of the five questions be averaged.

\section{Possible Weaknesses}

A very exhaustive evaluation may find some pitfalls in the performance of this system. In this section, some of these possible problems that could be found will be mentioned, as well as possible solution to them.

Rule implementation in the OC has been made exactly as the rules have been defined by physicians. Most of these rules work as expected in regular ovulatory women, as well as for some women with some irregularities in their cycles. However, in some special cases a rule may work with some extra criteria provided by the physicians. This additional criteria needs to be, first, well defined, and then implemented in the OC.

If after a comprehensive evaluation, it is proven that the system is useful, but too difficult to use by the medical personnel, there are Several possible ways to solve this problem:

- Improve the user interface capabilities.

- Implement some effective training lessons.

- Write a more understandable user manual.

Some physicians may find the $\mathrm{OC}$ a not very useful system or may want the OC with more features. The $O C$ has been implemented in an environment that is easy to expand, not only in the number of ovulation variables, but also in features, such as the 
type of information provided to the user, or the way results are presented.

Reliability of the decisions made may be questioned not because the whole system is unreliable, but because of one of the variables implemented. Therefore, it is strictly necessary to isolate the main source of unreliability, and implement another evaluation study for the rest of the system.

Confusion in the interpretation of the OC's decisions may result as a consequence of the way results are displayed. Knowing exactly the way physicians want the results to be displayed will help improve the interpretation of decisions in the OC.

This study may also show that the reference method could be biased because patients may be influenced by the information provided by the Ovustick. An alternative solution to that problem is to use serum LH analysis or ultrasound as a reference method. It would not be very convenient for the patients, because they will need to go to the clinic many days a month, but in the experimental group the patients have to make visits to the clinic anyway. Moreover, before patients are assigned to one of the groups, they have already agreed to the option of making clinic visits.

\section{$\underline{\text { Evaluation of Other Possible Features }}$}

There are other functions in the $\mathrm{OC}$ that have not been thoroughly evaluated. These are two of the most important features that are recommended to be tested in future evaluations.

- Software evaluation. A study has to be developed to find possible "bugs" in the whole program.

- User interface. This system has to be exposed to real life. Issues, such as instructions, help menus, and simplicity would be improved if the system is criticized by experts. 


\section{CHAPTER XII}

\section{CONCLUSIONS AND FUTURE RESEARCH}

In this chapter the OC's main features and contributions will be evaluated. This analysis has been made based on individual aspects in which, based on several studies, there were major contributions. A summary of some of these benefits is that this system provides rule comparison and evaluation, and predicts and detects the possible day day of ovulation. Also, this system is knowledge based, has decision-making capabilities, and keeps historical records for future analyses. It also combines more than one method for the diagnosis, is expandable, and is user-friendly. It is relatively cost-effective, is based on simple measurements, and provides the user choices of methods.

- The ability to compare and/or evaluate methods for the timing of the day of ovulation is probably the greatest achievement of this system. The lack of tools for this type of evaluation has been the main motivation in order to look for more objective tools. As long as the rules can be represented in an algorithm, the computer will be able to evaluate each rule for each single set of data or for many if desired. The OC's capabilities for handling data different types of data (e.g. temperature and resistance) allows comparison of methods based on different type of variables. To compare different type of variables, data has to be taken concurrently for each variable so that the results (e.g. the detection of the day of ovulation) may be compared. In that way, comparison of the results may lead to important conclusions concerning accuracy and reliability.

The concept of a computer-based system for evaluation and comparison rules con- 
cerning the ovulation timing is a new concept not implemented by anybody before. Although for years it has been evident the need for techniques to evaluate rules [4, 9], nobody has developed a computer-based system for such a purpose. As shown in Chapter IX, many important comparison results were obtained. Some of the results concerning accuracy, and aproximate number of day after ovulation had already been reported in the literature $[11,12,16]$. However, comparative studies as obtained by the OC are not usually available. Generally, evaluations of methods to detect the ovulation are based on a single reliable variable, such as $\mathrm{LH}$ hormone analysis. This, however, has led to a great diversity of results, many of them often not in agreement. As a result it is expected that a system such as the OC may have great acceptance as a more objective tool for comparison and evaluation of different methods.

- This system has also the ability to keep historical records of data taken either directly from the patient, or from charts kept in the past. As the amount of data grows, the potential ability to make statistical analyses and comparisons from the accumulated data will be very valuable and will contribute to important studies. This ability has long been desired by NFP researchers, whose data is occasionally incomplete because of some lost charts or values not recorded. In addition, the difficulties presented in analyzing historical records has discouraged retrospective studies in this field [8].

- Most of the current instruments or home kits utilized to determine the fertility status in women are based on only one method or parameter, e.g., temperature, ultrasound, LH assays, etc. This has shown to have inherent limitations, especially in women who have frequently irregular cycles. Some of these irregularities usually are independent of the method used. For example, irregularities in the temperature values may not be detected by the VER or SER techniques. Besides, it has been 
well accepted by physicians that the combination of two or more methods increases greatly the accuracy of the diagnosis $[5,11]$. For these reasons, the OC has included, in its primary stage, three numeric variables and many symptoms that have proven to be accurate indicators of the ovulation conditions. Therefore, a great improvement in reliability and accuracy is expected.

- Most reliable methods currently used to determine the ovulation day are based on sophisticated, expensive and complicated techniques. In some of these approaches, such as ultrasound or LH serum assays, patients are required to make many consecutive visits to the clinic every month. Also, some of these methods have problems related to comfort and convenience. In response to these issues, the $\mathrm{OC}$ has based its main parameters on relatively simple and reliable methods which, for the most part, do not present the problems mentioned above.

Let us consider the BBT method. This method has been proven to be one of the most popular and easiest methods of keeping track of ovulation. It has also been demonstrated that BBT is not an accurate predictor of ovulation; however, its potential as a reliable detector of ovulation, and therefore, of the luteal phase, has been widely accepted. On the other hand, some of the BBT's problems as a birth control method, reported by some physicians, are related to the inaccuracy of thermometer readings, poor charting techniques, or the difficulty of chart interpretations [9]. It is clear that the OC's programmed features eliminate some of these problems, making the method easier to interpret and more reliable. As long as the data has been gathered correctly, a process which is also assisted by the OC, human errors in the interpretation of the data are greatly reduced.

Now let us examine the VER and SER technique. This is one of the most recent methods developed for the prediction and detection of ovulation. Because of that, there is still some lingering doubt about its usefulness. However, many researchers 
have already published positive evaluations and have considered it to be a very precise and safe method. The special functions in the $\mathrm{OC}$ for the interpretation of these two variables simplify the decision making task.

- As mentioned in Chapter IV, there are many methods and devices used to confirm the day of ovulation. However, no devices have been built so far for the prediction of the time of ovulation. By using the SER variable measured by the CUE Monitor, the $\mathrm{OC}$ will be able to predict the day of ovulation 7 to 5 days in advance. This is a unique feature that has not been implemented before in any ovulation device. On the other hand, the detection of ovulation has been improved by detecting it by more than one method, e.g., BBT, VER, and symptoms. This will greatly assist fertile and subfertile couples who want to maximize the chance of conception when pregnancy is desired, or to special treatments, such as donor insemination, where ovulation timing is more critical.

- The $\mathrm{OC}$ is a knowledge-based system which has the ability to communicate with and guide the user, e.g., a nurse or physician. To do this, the $O C$ has been given well accepted rules for decision-making tasks. Unless the user wants to extract additional information from the data, no additional calculations are required to interpret data or make a decision. For the cases in which the decision is not that evident, the $\mathrm{OC}$ assists the user by providing results from different rules applied to the same set of data, which will hopefully narrow down the ambiguities in the decision. Also, it has been proven that there are many different patterns of cycle behavior even among normally ovulating women. By having more than one rule for each parameter measured, physicians will have wider perceptions of the changes in specific situations, especially in some of those cycles where ovulation could be detected by only one rule.

- Expandability has been one of the main concerns since the beginning of the 
development of this system. As defined before, the OC system bases its results mainly on three numeric variables, primarily because current studies have given them much credibility. However, if one or more new variables were to be added, minimum changes to the database would be required. It is common to see how some methods become obsolete or are no longer emphasized by gynecologists or other medical researchers. Indeed, the medical field keeps changing emphasis very rapidly. Therefore, the success of any medical system depends greatly on its adaptability and ease of modification so that any new variable, method, or variable extension may be added at any time without major changes to the system. This principle has been carefully considered since the beginning of this project. From this perspective, the OC has great potential as an ovulation monitor, since it presents a general database structure which is able to be increased to handle as many variables as desired.

Expandability in the OC is provided by both software and hardware. From the point of view of the hardware, the system already contains 5 extra analog inputs. Some modifications to the program that controls the ADC will need to be made. From the point of view of the software, 1-2-3 offers many possibilities for growth and modification of the database structure. The only changes or additions needed are concerned with rule implementation, which in this case is well assisted by the 1-2-3 command language. Some of these changes could be made by somebody who has some knowledge of the 1-2-3 spreadsheet. Other aspects of the system such as the database, data manipulation and the user interface techniques will remain the same.

- File storage and retrieval is undoubtedly one of the most important functions, especially when valuable data is being handled. These two functions have been carefully implemented to guarantee that mistakes made while saving data will not affect data already saved in the system. If the data has not been saved, there are some 
reminders that warn the user. Retrieval is direct and simple. Another aspect of data storage is the advantage of having separate files for each patient. This will help organize and maintain the data.

- From the point of view of the patient, there are also some useful features in the OC. First, the system is flexible, allowing more than one choice for those patients who may not wish to use one particular method. Second, not only does the patient obtain immediate benefits by knowing her fertility status, but also she automatically starts accumulating data that could be extremely useful for future studies about any particular abnormality in her fertile life. This type of benefit is very difficult to obtain based on traditional charting methods.

- Although physicians or nurses will make the final decision about fertility conditions in the patient, the automatic diagnosis features included in the $\mathrm{OC}$ will be well appreciated by them. The time they will spend in making a regular diagnosis should be much less than that using traditional methods. This should improve the efficiency of the personnel. For some specific studies in which doctors may need to review historical records about the patient, the OC could also save a great amount of time.

- Cost and maintenance of the system is another attractive feature of the OC. The whole system is based on standard hardware and software. The use of a 286,386 or 486 compatible microcomputer provides different options to clinics or hospitals, depending on the number of patients and the allotted budget. The fact that 1-2-3 is one of the most commonly available spreadsheets for personal computers reduces the cost of software for those hospitals or clinics that have already bought it. In addition, the fact that this system is built based on an IBM-compatible microcomputer, support will be available to take advantage of potential improvements based on faster and more powerful microcomputers. The analog-to-digital converter 
selection is less flexible, in the sense that there are no standards stipulated for ADC's. ADC's also depend on each individual need. They could be 8 or 12 or 16bit resolution, have from 1 to 16 or more analog inputs, and have a variety of conversion times. Our system recommends at least a 4-input, 12-bit ADC, whose conversion time is less than $100 \mathrm{msec} / \mathrm{channel}$.

- Using 1-2-3 as the main spreadsheet presents also many advantages. First, 1-2-3 has guaranteed compatibility with previous releases since its Release 1.A in 1982. Although the $O C$ only runs under Release 3.1, future releases will also guarantee compatibility with the Release 3.1. Second, there has lately been a growing interest in spreadsheet applications among professionals in the field of medicine. Physicians or nurses with some knowledge of 1-2-3 could exploit many more spreadsheet functions that were not included in the OC macros.

- Another important goal from the beginning of this project was the design of a userfriendly system. 1-2-3's custom menus are perhaps the most useful functions to create easy-to-use interactive programs. For the most part, mouse buttons, or alternatively the arrow and enter keys, are all that the user needs to execute the commands. On-line help is provided for each menu level. Critical feedback from medical experts concerning improvements in user interface features is also necessary. If such modifications are required, a Windows environment (e.g., MS-Windows 3) might be useful. That would greatly enhance the user interface, allowing more visual fearures and on-line help menus.

- Although the original goals were fulfilled, this system is still in its beginning stages and some future research and improvements are necessary. First, the system should be exposed to medical practitioners in this field and the feedback obtained from these experts used to optimize the system. Second, the system needs to be given more medical terms. So far, the OC may have many of terms which make no sense 
to a physician, or that may be interpreted wrongly, but this requires expertise in the medical field, which is outside the scope of this project. Third, in conjunction with the two aspects mentioned above, this system needs to be tested more thoroughly, with data from large numbers of different women. The test done so far has been theoretical in nature, although taken from very reliable sources, but the $\mathrm{OC}$ needs to be evaluated in a wider variety of special cases. Some of these improvements to the system can be made by the user himself, since most of the rules can be partially modified or tuned up by using the proper commands explained in the user manual. Physicians may also suggest the addition or modification of some of the rules. Not all physicians agree as to the most reliable and accurate methods to be used, and therefore, the $\mathrm{OC}$ has been given great flexibility which will allow this system to be individualized by the researchers using it.

- Obviously, the interface hardware between the $\mathrm{A} / \mathrm{D}$ converter and the electrical leads of the sensors needs to be implemented. This hardware is strictly dependent on the type and brand of sensors selected. Therefore, the first step is to purchase the sensor and their technical information, and then adapt them to the $A / D$ converter. Some of the signal conditioning could be done both by hardware or by software. For example, linearlization or scaling could be done by a program.

- Finally, the system as developed has a solid base on which to continue to build more complex features. It automates data management, provides data display, collection, manipulation, storage, and graphic depiction. This system has been developed for the medical field, since there is an obvious need for such a system. Theoretical concepts from the decision-medical support systems have been used in its implementation. Concepts about monitoring electrical signals or keeping track of industrial processes have also been implicitly used in this system. The use of standard, relatively inexpensive hardware and software tools, many of which may be already be 
owned by most companies and institutions, may encourage more research into stand-along systems with data visualization, and decision-making capacity in nontraditional systems of our society. 


\section{REFERENCES}

[1] Brown JB, Blackwell LF, Billings JJ, Conway B, Cox RI, Garrett G, Holmes J, and Smith MA: "Natural Family Planning" Obstet Gynecol 157:1082-9, 1987.

[2] Vermesh M, Kletzky OA, Davajan V, and Israel R: "Monitoring techniques to predict and detect ovulation." Fertil Steril 47: 259-64, 1987.

[3] Batzer FR: "Ultrasonic indices of ovulation." J Reprod Med 31:764, 1986.

[4] Campbell KL: "Methods of Monitoring Ovarian Function and Predicting Ovulation: Summary of a Meeting." Research Frontiers in Fertility Regulation Vol 3 N5, 1985.

[5] Kenigsberg D: "New tests for the prediction of ovulation." Clin Obstet Gynecol 32:533-40, 1989.

[6] Perkowski M, Kruszynki H, Fijalkowski W, and Kulpa K: "A Multi- Parameter Microprocessor Based Ovulation and Fertility Predictor/ Indicator", Memorandum and grant proposal given to Pope John II, Vatican Library, May 1981.

[7] Hatcher RA, Stewart F, Trussell J, Kowal D, Guest F, Stewart GK, and Cates W: "Contraceptive Technology 1990-1992" Irvington Publishers, Inc., New York, 1990.

[8] Michael PA, Kanich RE, Hall CP, and Ruchte SH: "Computerized clinical histories: The development of an HIS subsystem in a community hospital." Proceedings of the Third Annual IEEE Symposium on Computer-Based Medical Systems. June 3-6, 1990. Chapel Hill, NC. p. 462-468.

[9] McCarthy JJ and Rockette HE: "A comparison of methods to interpret the basal body temperature graph" Fertil Steril 39:640-6, 1983.

[10] McCarthy LL, and Rockette HE: "Prediction of Ovulation with Basal Body Temperature", The Journal of Reproductive Medicine 31:742- 747, 1986.

[11] Moghissi KS: "Prediction and detection of ovulation." Fertil Steril 34:89-98, 1980.

[12] Huneger RJ and Fuller R: "A couple's guide to fertility: The complete symptothermal method", Northwest Natural Family Planning Services, 1986.

[13] Pukuslian L: "Salivary electrolyte changes during the normal menstrual cycle." $J$ Dent Res 51:1212, 1972.

[14] Manusco S, Bellante FP, Morana R, and Angelini A: "Chemical changes in saliva during the menstrual cycle." Acta Med Rom 16:387, 1978.

[15] Moreno JE, Weitzman GA, Doody MC, Gibbons WE, Besch P, and Goldzieher JW: "Temporal relation of ovulation to salivary and vaginal electrical resistance patterns: Implications for natural family planning." Contraception 38:407-18, 1988. 
[16] Albrecht BH, Fernando RS, Regas J, and Betz G: "A new method for predicting and confirming ovulation." Fertil Steril 44:200-205, 1985.

[17] Fazleabas AT, Segraves MM, and Khan-Danwood FS: "Evaluation of salivary and vaginal electrical resistance for determination of the time of ovulation." Int J Fertil 35:106-11, 1990.

[18] Fernando RS, Regas J, Betz G: "Prediction of ovulation with the use of oral and vaginal electrical measurements during treatment with clomiphene citrate." Fertil Steril 47:409-15, 1987.

[19] Roumen FJ, Dieben TO: "Ovulation prediction by monitoring salivary electrical resistance with the Cue Fertility Monitor." Obstet Gynecol 71:49-52, 1988.

[20] Jacobs MH, Blasco L, Sondheimer SJ: "Ovulation prediction by monitoring salivary and vaginal electrical resistance with the PEAK Ovulation Predictor." Obstet Gynecol 73:817-22, 1989.

[21] Fordney-Settlage D: "A review of Cervical Mucus and Sperm Interactions in Humans." Int J Fertil 26:161-169, 1981.

[22] MacDonald RR, and Lumley IB: "Endocervical pH Measured in Vivo Through the Normal Menstrual Cycle." Obstet Gynecol 35:202-206, 1970.

[23] Moghissi KS: The function of the cervix in fertility." Fertil Steril 23:295-305, 1972.

[24] Ismail M, Arshat H, Pulcrano J, Royston P, and Spieler J: "An evaluation of the BIOSELF 110 fertility indicator." Contraception 39:53-71, 1989.

[25] Calamera JC, Vilar O, Nicholson R: "Changes in sialic acid concentration in human saliva during the menstrual cycle." Int J Fertil 31:43-5, 1986.

[26] Laurie SL: "Periodic Abstinence." Population Reports Series I, No. 3, September, 1981.

[27] Sugiyama S: "L-Sophia. Questions \& Answers", Japan, 1990.

[28] Rodriguez MH, Mishell DR Jr, Grozinger C, Cao JN, Lacarra M: "Comparison of TestPack and Ovustick for predicting ovulation." J Reprod Med 35:133-5, 1990.

[29] Daniel JC Jr, Lonergan PB, Sullivan PK, Taylor SP: "Evaluation of "Determine: The OvuTest" as a device for identifying optimal time for conception." Fertil Steril 47:590-6, 1987.

[30] Labrecque M, Drouin J, Rioux JE, Gingras S, Spieler JM: "Validity of the Bioself 110 fertility indicator." Fertil Steril 52 :604-8, 1989.

[31] Fumai C, Collet C, Petroni M, Malowany AS, Carnevale FA, Gottesman RD, Rouseau A: "The design of a simulator for an intensive care unit Patient Data Management System." Proceedings of the Third Annual IEEE Symposium on Computer-Based Medical Systems. June 3-6, 1990. Chapel Hill, NC. p. 454-461.

[32] Mart RR: "In-service use-effectiveness analysis using a microcomputer AT Action Familiale in Mauritius" Abstracts of papers presented at the IVth Congress of the International Federation for Family Life Promotion Ottawa, Canada, June-July, 1986. 
[33] Butko SN and Ol'shanskii VK: "New Decision Support Systems in World Medicine", Scientific-Industrial Organization of Automatic Control Systems Moscow, USSR, June, 1990.

[34] Shortliffe EH: "Computer Programs to Support Clinical Decision Making", JAMA 258:61-66, 1987.

[35] Barnett GO, Cimino JJ, Hupp JA, and Hoffer EP: "Dxplain. An evolving diagnostic decision-support system", JAMA 258:67-74, 1987.

[36] Burr-Brown: "The Handbook of Personal Computer Instrumentation", 1990.

[37] Real Time Devices, Inc. "PC Bus Data Acquisition and Control Product Guide", Vol. 3, No. 2.

[38] Hilgers TW, and Bailey AJ: "Natural Family Planing. II. Basal Body Temperature and Estimated Time of Ovulation", Obstet Gynecol 55:(3)333-339, 1980.

[39] Thornton SJ, Peperell RJ, and Brown JB: "Home Monitoring of Gonadotropin Ovulation Induction Using the Ovarian Monitor", Fertil Steril 54:1076,1082, 1990.

[40] Baker HW, Burger HG, Kovacs GT, Summerbell D, and Wames GM: "Timing of ovulation by determination of the urinary luteinizing hormone surge with an enzyme-linked monoclonal antibody dipstick (Ovustick)", Aust NZ J Obstet Gynaecol 26:79, 1986.

[41] Lotus 1-2-3 Release 3.1: Reference Manual, 1990. 


\section{GLOSSARY}

Abstinence: Refraining from sexual intercourse.

Amenorrhea: Prolonged absence or suppression of menstruation. This state is normal before puberty, after menopause, and during pregnancy and lactation.

Anovulatory: Without ovulation (a cycle without ovulation is anovulatory).

Antibody: An immunoglobulin molecule having a specific amino acid sequency by virtue of which it interacts only with the antigen that induced its synthesis in lymphoid tissue, or with antigen closely related to it.

Antrum: The dilated portion of the pyloric part of the stomach, between the body of the stomach and the pyloric canal.

Artificial insemination: Introduction of semen into the uterus or oviduct by other than natural means.

Basal Body Temperature (BBT): The temperature of the body at rest, unaffected by activity; taken upon waking ("waking temperature").

Basal Body Temperature Method: (BBT) A method of contraception that uses daily temperature readings taken immediately after waking to identify the time of ovulation; approximately 24 hours after ovulation the BBT increases.

Biological Assay: Bioassay. Determination of the amount of a particular constituent of a mixture.

Biphasic: Two different phases, as in biphasic basal body temperature which shows a rise in temperature in the second half of the cycle suggestion ovulation has occurred.

Cervical Mucus: A mucous fluid secreted by crypts in the cervix.

Cervix: The lower, narrow part of the uterus-it opens at birth to provide a birth canal, and has mucus-producing "crypte".

Conception: Generally the beginning of pregnancy. Conception is usually equated with the fertilization of the ovum by the sperm but is sometimes equated with the implantation of the fertilized ovum in the uterine lining.

Contraception: Interference with reproductive physiology or with the act of sexual intercourse in order to prevent conception. "Contraception" is to be distinguished from "Natural Family Planning" (which relies on natural fertility awareness and periodic abstinence when avoiding pregnancy) and from "Abortion" (destroying human life 
after conception to prevent birth).

Corpus Luteum: A gland which for 10-14 days after ovulation secretes the hormone progesterone. This gland is the remainder of the follicle (housing) from which the egg was released at ovulation, and it is yellow in color; hence, the name "corpus luteum" (latin for "yellow body").

Egg: An ovum; a female gamete; an oocyte; a female reproductive cell at any stage before fertilization.

Enzyme: A protein capable of accelerating or producing by catalytic action some change in a substrate for which it is often specific.

Estrogen: A female hormone partly responsible for cyclic changes in the female reproductive system (unopposed estrogen causes the cervix to secrete mucus). It is also responsible for secondary sex characteristics in the woman.

Fallopian Tube: A tube, with a trumpet-like end, to receive and conduct the egg from either ovary to the uterus.

Fertile: Capable of conceiving a child.

Fertility Rate: (also referred to as the general fertility rate) The number of live births per 1000 women aged 15 to 44 years in a given year.

Follicle: Any one of thousands of tiny containers each holding an ovum (egg). Upon releasing its ovum it becomes a gland called the "corpus Luteum". One type of follicle is an ovarian follicle which is a very small sac in the ovary in which an ovum matures and from which the egg is released.

FSH: Abbreviation for "Follicle Stimulating Hormone," released by the pituitary gland in the brain to stimulate maturation of follicles in the ovary.

Glucose: In certain foodstufs, especially fruit, and in normal blood, the chief source of energy for living organisms.

Hypothalamus: Part of the brain just above the pituitary which helps regulate basic functions such as sleep, appetite, body temperature, fertility. The hypothalamus in influenced by higher cortical levels of the brain and controls hormone production by the pituitary.

Hormone: A chemical substance produced in the body which has a specific regulatory effect on the activity of certain cells or a certain organ or organs.

Implantation: The process by which a newly conceived human being at the blastocyst stage imbeds itself in the lining of the uterus about 6-8 days after conception.

Immunoassay: The measurement of antigen-antibody interaction, as by immunofluorescent techniques, radioimmunoassay, etc.

In vitro fertilization: A procedure in which an egg is removed from a ripe follicle and fertilized by a sperm cell outside the human body. The fertilized egg is allowed to 
divide in a protected environment for about two days and then is inserted back into the uterus of the woman.

Infertility: The inability in a woman to conceive or in a man to fertilize an egg.

Lactation: Producing and yielding milk from the mammary glands (the breasts). Lactation can produce anovulation.

Leuocyte: White cell; a colorless blood corpuscle capable of ameboid movement, whose chief function is to protect the body against microorganisms causing disease.

LH: Abbreviation for "Luteinizing Hormone," released by the pituitary gland in the brain to trigger ovulation.

Luteal Phase: The time (normally 10-14 days) of significant progesterone secretion from the luteinized follicle or post-ovulatory corpus luteum before menses.

Menarche: The first menstruation (may occur before of after the first ovulation).

Menopause: The permanent cessation of menstruation and ovarian activity.

Menses (Menstruation): A blood and mucus discharge consisting of the sloughed-off outer layers of the uterus's inner lining (see "True Menstruation").

Monoclonal: Derived from a single cell; pertaining to a single clone.

Mucin: A mucopolysaccharide or glycoprotein, the chief constituent of mucus.

OS ("os", Latin for "mouth"): The opening, or "mouth" of the cervix.

Ovary: The almond-shaped female reproductive organ containing the ova (eggs).

Ovulation: The process by which an ovum (egg) is released from its follicle (housing) through the wall of the ovary, making a woman temporarity fertile.

Ovulation Method ("OM"): The "Ovulation Method" of NFP developed by Drs. John and Lyn Billings of Australia to determine times of infertility and possible and probably fertility by exclusive reliance upon sensed and/or seen vulval manifestations of the absence, presence, traits, and changing or unchanging character of vaginal discharge, particularly of cervical mucus. The World Organization of the Ovulation Method/Billings (WOOMB) was begun in 1977 to promote fertility awareness and family life through natural family planning according to the Billings Method. Dr. Thomas W. Hilgers developed the Creighton Model of OM education based on the tissue-paper exam.

Ovum (plural "ova"): The woman's egg.

Peak Day: The final day of any sign of any trait of the "Most Fertile Sign" during a possibly fertile time. During a typical mucus pattern the most fertile sign is EW-M; during a patch, M; during spotting, any bleeding; and during a prolonged discharge, any "difference" that may appear in the discharge. After each such "peak" fertility is assumed for the next four days (until evening of the fourth), in case ovulation was 
associated with it. If there is more than one peak in a cycle, the peak accompanied by a sustained temperature rise is considered the presumptively ovulatory peak of the cycle.

Pearl index: The number of pregnancies per 100100 per year (100 woman-years) exposure, usually used as a measure of contraceptive failure.

Periodic Abstinence Methods: Contraceptive methods that rely on timing of intercourse to avoid the ovulatory phase of a woman's menstrual cycle; also called fertility awareness or natural family planning. 1. The basal body temperature (BBT) method uses daily temperature readings to identify the time of ovulation. 2 . In the ovulation of Billings method, women identify the relationships of changes in cervical mucus to fertile and infertile days. 3. The symptothermal method charts changes in temperature, cervical mucus and other symptoms of ovulation (i.e., intermenstrual pain).

Pituitary: Pituitary gland.

Post-ovulatory: The time of the cycle during which ovulation can no longer occur.

Pre-menopause: The time (usually the final 60 cycles or so) before menopause, during which the menstrual cycle pattern may change to reflect irregular, decreasing fertility by greater variation in cycle lengths, altered menstrual and cervical mucus patterns, fewer thermal shifts, and shorter luteal phases.'

Pre-ovulatory: The time during the cycle prior to the occurrence of ovulation.

Pre-rise Base: The highest undisturbed temperature of the final six "low" readings before the "thermal shift" (temperature rise). The amount of "rise" is measured from the pre-rise base.

Progesterone: A female hormone secreted during the luteal phase to prevent further ovulation while enriching the uterine lining for possible implantation. it also causes the basal body temperature to rise and the cervical mucus to dry up.

Progestins: A large group of synthetic drugs that have a progesterone-like effect on the uterus.

Radioimmunoassay: Immunoassay using a radioactive-labeled substance that reacts with the substance under test.

Rhythm Method (Calendar Rhythm): A system of calculating the times of fertility and infertility in the present cycle on the basis of the length of previous cycles and certain assumptions about the time of ovulation and sperm survival. Rhythm was based on the independent but simultaneous discoveries of Ogino in Japan and Knaus in Austria in the late 1920's, that the post-ovulatory phase of the cycle is "constant" in length. It was gradually superseded by discoveries of specific hormonally-based signs of the possibly fertile time (the temperature, mucus, cervix, and secondary signs).

Serum: The clear portion of any liquid separated from its more solid elements. 
Semen The thick, whitish fluid which normally contains sperm and seminal secretions and is ejected during ejaculation. It causes conception when it unites with a woman's egg.

Spermatozoon: A mature male germ cell, the specific output of the testes, which impregnates the ovum in sexual reproduction.

Spinnbarkeit: A test to determine cervical mucus viscosity. A thread of cervical mucus is stretched between two glass slides and its length is measured. The time at which it can be drawn to maximum length (lowest viscosity) usually precedes or coincides with the time of ovulation.

Sympto-Thermal Method (STM): Any natural family planning system making use of all the signs of fertility: basal body temperature, cervical changes, cervical mucus, and various secondary signs.

Symptom: Subjective evidence of disease or the condition of the patient.

Thermal Shift: The change in basal body temperature level from early cycle lows to late cycle highs. The World Health Organization (WHO) considers the shift to be "significant" for defining infertility after ovulation when the temperature rises within two days to "full thermal shift level" $\left(0.36^{\circ} \mathrm{F}\right.$ or more above the highest of the final 6 lows) and stays there for three full days. The Sympto-Thermal Method requires crosschecking the temperature with cervix and/or mucus signs before assuming infertility, but because of the crosscheck, does not require such a pronounced temperature rise to evaluate the temperature pattern.

True Menstruation: Bleeding is only called "true" menstruation, for use with the 6-Day Rule, if the Basic Sympto-Thermal Rule was fulfilled prior to the onset of the bleeding.

Ultrasound: Sound echo images of soft internal structures. Also called ultrasonography, can be used to determine size and position of the fetus, the placenta, a developing follicle, or a tumor.

Uterus: The womb, in which the baby grows during pregnancy.

Vagina: The soft muscular tract into which the man's penis fits during intercourse. The vagina connects the cervix with the vulva and is a birth canal.

Vulva: The outer parts of the female sex organs, including the vaginal lips (labia). 